\title{
Comparación de los patrones espaciales e incidencia de la segregación residencial en las principales ciudades de Colombia
}

\author{
Comparison of spatial patterns and incidence of residential segregation \\ in the main cities of Colombia
}

\author{
José Mario Mayorga Henao ${ }^{1}$ (B)
}

\begin{abstract}
Resumen
El artículo tiene como objetivo principal describir los patrones geográficos y la incidencia de la segregación residencial en Bogotá, Cali y Medellín en los años 2005 y 2018. A partir de una reflexión conceptual y metodológica sobre la importancia de hacer estudios comparativos en tiempo y contexto, se hace un planteamiento para medir la segregación residencial en las tres principales ciudades de Colombia en dos años diferentes y a diversas escalas para poder comparar cómo ha sido la incidencia de la segregación residencial y los cambios espaciales que ha presentado. Para ello se usan múltiples métodos de análisis espacial y estadístico que permiten la construcción de índices a dos escalas que llevan a la conclusión que la segregación presenta particularidades en sus patrones geográficos en las tres ciudades estudiadas. Entre las particularidades se encuentra que la segregación ha disminuido a escala macro, se presenta con mayor incidencia a una escala barrial y que hay una baja probabilidad de interacción entre los grupos de calidad de vida baja y calidad de vida alta.
\end{abstract}

Palabras clave: segregación residencial; indicadores espaciales de segregación; ciudades colombianas; patrones espaciales.

\begin{abstract}
This article describes the geographic patterns and incidence of residential segregation in Bogotá, Cali, and Medellín in the years 2005 and 2018. Starting from a conceptual and methodological reflection on the importance of making comparative studies in time and context, a methodological approach is proposed to measure residential segregation in the three main cities of Colombia in two different years and at different scales. The incidence of residential segregation is compared as well as the spatial changes that it has caused. For this, multiple methods of spatial and statistical analysis are used that enable the construction of indices at different scales that lead to the conclusion that segregation presents particularities in its geographical patterns in the three cities. Among the particularities, it is found that segregation has decreased on a macro-scale, but has an increased incidence on a neighbourhood scale, and that there is a low probability of interaction between the poor and rich.
\end{abstract}

Keywords: residential segregation; spatial indicators of segregation; Colombian cities; spatial patterns.

\section{Introducción}

La investigación sobre cómo se distribuyen los grupos sociales en el espacio urbano ha generado múltiples discusiones dentro de las ciencias sociales desde principios del siglo XX con el surgimiento de la reflexión en la escuela de Chicago. El concepto de segregación es usado de manera sociológica por

1 Departamento de Geografía, Universidad Nacional de Colombia, Colombia. jmmayorgah@unal.edu.co 
primera vez por la escuela de Chicago, en cabeza de R.E. Park y E.W Burgess, en sus ensayos de 1925 y 1929. Para estos autores, la segregación es el resultado de una concentración natural de la población urbana, por lo cual las áreas de segregación son productos de las fuerzas sociales y las necesidades económicas. En su concepto, la ciudad selecciona y segrega a la población de manera natural, y así cada grupo ocupa su espacio (Park, 1999).

A partir de los aportes hechos por la escuela de Chicago, el concepto de segregación ha tenido diferentes aproximaciones desde las ciencias sociales. La sociología, por ejemplo, hace énfasis en la presencia o ausencia de interacción entre diferentes grupos sociales; por su parte, la geografía en términos generales ha centrado el debate en la desigualdad en la distribución de los grupos sociales en el espacio físico, y la economía ha analizado con mayor énfasis el impacto de las rentas del suelo en el acceso a la vivienda (Salas, 2008). White (1983) indica que estos tipos de segregación se pueden mantener separados conceptualmente, pues no se implican uno al otro en un contexto específico. Plantea entonces que se pueden presentar correlaciones dado que la ausencia de interacción entre grupos sociales se puede expresar espacialmente en una desigual distribución.

En términos generales, hay un consenso en torno a que segregación residencial se refiere al grado en el cual dos o más grupos sociales diferencian entre sí sus lugares de residencia en el espacio urbano, noción a la cual se le atribuyen distintos grados de complejidad que se reflejan en la variedad de índices propuestos para su medición (Massey \& Denton, 1988). La segregación residencial, se manifiesta por relaciones de proximidad o distancia residencial entre diferentes grupos, lo cual genera tendencias de homogeneidad o concentración de zonas de la ciudad que tienden a imposibilitar el contacto entre grupos (Rodríguez y Arriagada, 2004).

Si bien hay una definición general del fenómeno, Ospino (2017) resalta la importancia del proceso histórico y del contexto particular en el que se manifiesta. Al respecto Maloutas (2012) hace énfasis en que bajo el concepto de segregación se engloban múltiples procesos que no responden a una explicación universal. La segregación residencial como proceso y puesta en contexto, se relaciona con la conformación de patrones espaciales en los que interactúan de manera compleja el mercado de vivienda, el estado, la cultura y las características del territorio.

Se ha identificado que hay diferencias claras en la definición de los grupos sociales para la investigación de la segregación residencial en occidente. Por un lado, en Latinoamérica, se plantea que el principal motivo asociado a la segregación residencial es el factor socioeconómico, a diferencia por ejemplo del contexto estadounidense, donde el tema racial asume la mayor relevancia (Jargowsky, 1997), o el contexto europeo, en donde la procedencia de los hogares resulta fundamental por dar cuenta del fenómeno migratorio (Cantle, 2001; De Esteban, Curiel y Perelló, 2003; Carrasco I, 2007).

De lo anterior se resalta la importancia de estudios comparativos que permitan generar datos sobre los elementos comunes y singulares de la segregación residencial en diferentes contextos y en diferentes momentos. Se resalta la importancia de una perspectiva comparativa para entender los cambios que se presentan en la segregación y su relación con los cambios en la estructura económica. Más recientemente Maloutas (2012) plantea que en la comparación del fenómeno es importante observar los diferentes grados de segregación, los diferentes patrones espaciales de distribución de los grupos, la incidencia del mercado y de las políticas públicas y los impactos diferenciales que se presentan. Dureau et al. (2015) insisten en que la investigación sobre fenómenos urbanos como la segregación no se puede quedar en la especificidad local y no tomar en cuenta lo que pasa en otros contextos, para así poder dar cuenta de las diferencias y las sinergias que se presentan en el territorio. Por último, Musterd (2020), introduce diferentes investigaciones sobre segregación haciendo énfasis en que es un fenómeno relevante en diferentes ciudades del mundo, por lo cual se deben resaltar las fuerzas comunes en la conformación de los patrones.

En el caso de la comparación del fenómeno en Latinoamérica, algunos autores han concluido que en la mayoría de las ciudades metropolitanas del subcontinente hay unos patrones geográficos similares que permiten la generación de tipologías de segregación (Griffin y Ford, 1980; Bahr y Mertins, 1982; Gormsen, 1991; Ford, 1996; Ortiz y Schiappacasse, 2000; Janoschka 2002; Borsdorf 2003).

Estos patrones geográficos han sido explorados en varias investigaciones a lo largo del continente, en las que se destacan realizadas en Santiago de Chile por Sabatini (2000) en Montevideo por Katzman (2005) y Artigas, Chabalgoity, Garcia, Mediana y Trinchitella (2002), en Caracas por Carialo y Locabana (2001), en Buenos Aires por Prevot (2002) y Janoschka (2002) y en México por Aguilar (2002). En los 
últimos años se pueden destacar las investigaciones realizadas por Merengo y Elorza (2014) y por Rodríguez (2014) en Argentina, por Vázquez (2018) en Uruguay, por Sabatini, Rasse, Mora y Brain (2012), Ruiz y López (2014) y Rasse (2015) en Chile y por Mier, Velásquez y Zicardi (2012) en México.

En Colombia, los estudios sobre segregación han presentado un relativo interés tanto desde la academia como desde la administración pública. En Bogotá, la segregación ha tenido un interés como objeto de estudio en los últimos 20 años. A propósito, Mayorga (2019) identifica cerca de 33 investigaciones realizadas sobre el tema en la ciudad, las cuales han sido realizadas tanto en la academia como como en la administración pública. Dentro de los más relevantes y más citados s se encuentran las investigaciones realizadas por la Secretaría Distrital de Planeación en los años 2007 y 2013, en las cuales se estima la magnitud del fenómeno a partir de los estratos ${ }^{2}$, teniendo como base a las localidades ${ }^{3}$.

En el caso de Salas (2008) se calcula un índice de clasificación social a partir de los años de educación de la población. Se resalta la investigación de Aliaga y Álvarez (2010), en la cual se infieren condiciones de calidad de vida y su distribución espacial a partir de los estratos socioeconómicos. Dureau, et al. (2015) desarrollan una investigación en la que clasifica los grupos sociales según un índice de condición social de los hogares (ICS) con tres variables de la información Censal y estima unos indicadores de disimilitud a escalas micro en Bogotá. Por su parte Alfonso (2017) genera unos resultados que indican que los hogares bogotanos tienen una propensión a pagar más cara la vivienda en zonas con menor densidad poblacional y mayor dotación de activos públicos.

Sin embargo, la investigación ha estado limitada y concentrada al estudio de la segregación en Bogotá, a pesar de la importancia del sistema urbano en Colombia en el que se resalta la existencia de múltiples aglomeraciones que superan el millón de habitantes (Montoya, 2013; Departamento Nacional de Planeación [DNP], 2014; León y Ruiz, 2016), es poca la atención que se la ha prestado a la segregación en otras ciudades.

En el caso de Cali, se identifica la investigación de Vivas (2013) en el que se concluye que en la ciudad se presenta un alto nivel de segregación de la población afrocolombiana. Loaiza y Carvajal (2014) obtienen como resultado, que en Cali hay sectores de la ciudad que puede ser calificadas como homogéneas en su composición socioeconómica, según el cálculo del Índice de Segregación Espacial y Socioeconómico.

En Medellín, Medina, Morales y Núñez (2008) formulan múltiples indicadores con base en el censo del año 2005 para ilustrar la segregación espacial en la ciudad. Por su parte, Velásquez (2012) identifica la relación entre el ordenamiento territorial y la segregación residencial durante el periodo 2006-2011, a partir de un análisis de la localización de los proyectos de vivienda de interés social.

De la revisión del estado de la investigación sobre segregación en Colombia se concluye que aún es un campo en el que se pueden realizar aportes significativos. Como se discutirá más adelante, en la investigación realizada en el país no hay comparaciones temporales y no hay comparaciones entre ciudades. Además de ello, no se evidencia una reflexión sobre la escala de medición de la segregación y sobre la clasificación de los grupos sociales, dado que en términos generales se asumen clasificaciones preestablecidas por las entidades públicas (como por ejemplo los estratos). Así mismo, no se usan indicadores de segregación que incluyan variables espaciales para su estimación.

Para superar los problemas identificados en la investigación sobre segregación en Colombia, se plantea que es un proceso socioespacial que se despliega a través de múltiples variables y en diversas escalas. De tal forma, se descarta que la segregación sea una condición estática de distribución de los grupos sociales en el espacio y, por el contrario, se resalta la necesidad de investigar sobre la segregación en diferentes momentos para establecer cómo son los los patrones geográficos de cambio en los diferentes contextos.

Con respecto a la medición del fenómeno, se resalta la necesidad de utilizar indicadores espaciales que partan de una reflexión de la escala de los datos. Junto con el uso de información que dé cuenta de las diferentes variables socioeconómicas para la clasificación de los grupos sociales, se hace necesario pensar en alternativas metodológicas para la medición de los procesos de segregación en las ciudades colombianas. Esto supone, además, la generación de indicadores comunes que permitan la comparación.

2 Índice elaborado por la administración de la ciudad en el que se evalúa la calidad de las viviendas y el entorno urbano, asignando un número de estrato del 1 al 6 a un grupo de viviendas que, por lo general, corresponde a una manzana: cuanto más alto sea el número, mayor alto es el estrato (Aliaga y Álvarez 2010).

3 División del territorio de Bogotá en 20 unidades administrativas para la gestión y ejecución de programas y proyectos locales. 
Considerando lo anterior, el objetivo central que subyace en el presente estudio es describir los patrones espaciales y la incidencia de la segregación en las principales ciudades del sistema urbano de Colombia entre el periodo 2005-2018.

\section{Metodología}

\section{1. Área de estudio}

El análisis propuesto comprende la estimación y descripción de los indicadores de segregación de las tres principales ciudades del país: Bogotá, Medellín y Cali. Estas tres ciudades, en conjunto aportan más del 30\% del PIB Nacional, y son los vértices del denominado Triángulo de Oro de Colombia.

Figura 1. Ciudades de estudio en Colombia

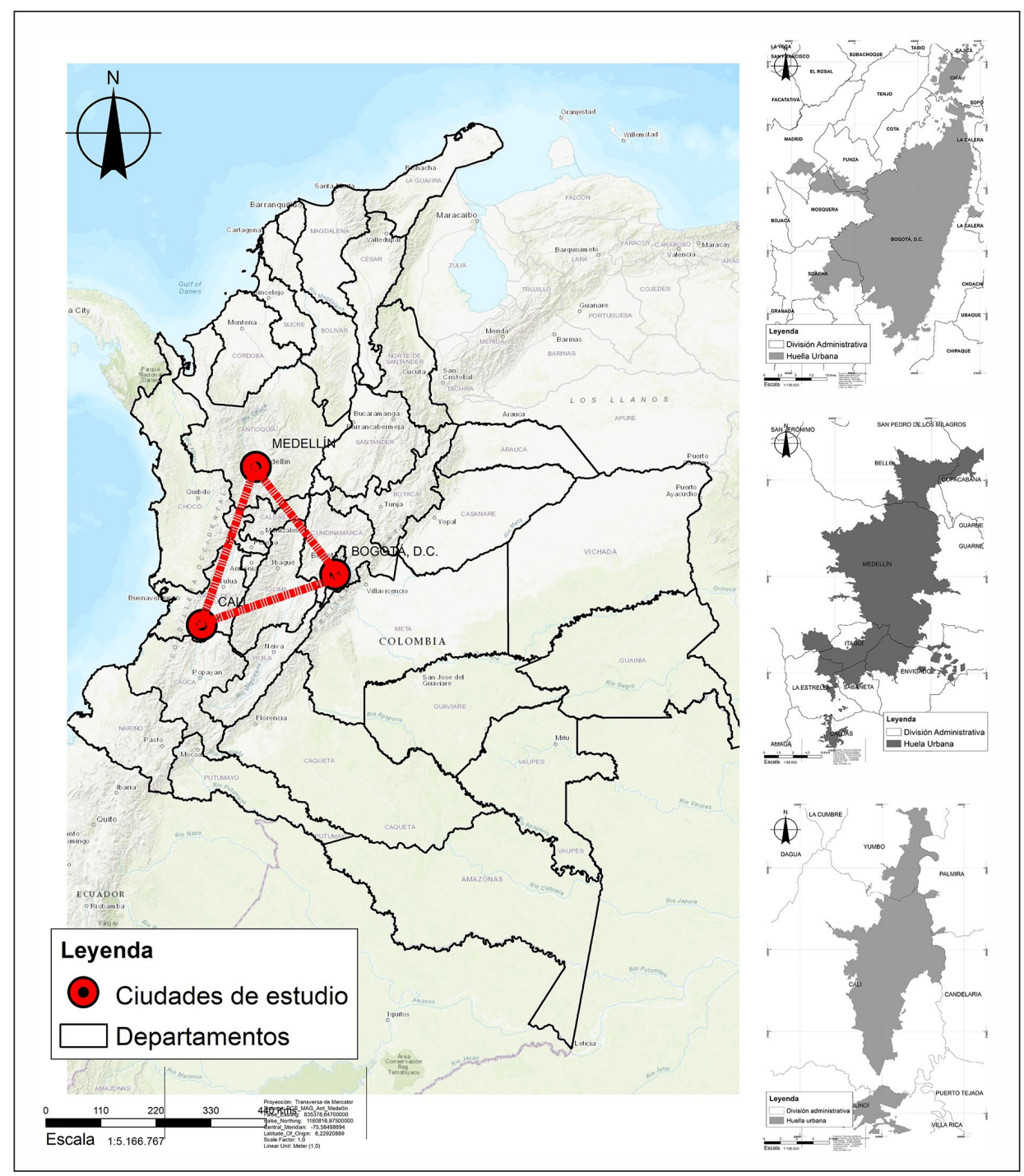

Elaboración propia

Las tres ciudades estudiadas han presentado intensos cambios espaciales durante los últimos treinta años, producto de relaciones funcionales y dinámicas de mercado que han llevado a que se rebasen sus límites político administrativos y se conformen aglomeraciones con otros municipios con los que comparten dinámicas funcionales y problemáticas comunes. Las tres ciudades de estudio se definen como aglomeraciones urbanas ya que presentan fuertes relaciones en torno a una ciudad principal o núcleo, que concentra dinámicas económicas, territoriales y poblacionales de mayor escala que aquellas con las que se relaciona, pero cuyo desarrollo incluye unos municipios secundarios. Esto lleva a la conformación 
de una ciudad principal, compuesta por diferentes nodos, cuya área rebasa los límites político-administrativos de la ciudad principal (DNP, 2014).

De tal forma, acorde con el marco conceptual propuesto, el ámbito de estudio no se reduce a las ciudades al interior de su límite político administrativo, como tradicionalmente se ha hecho la investigación en Colombia, sino que busca reconocer la segregación de manera amplia vinculando la distribución de los grupos sociales en todo el continuo urbano de las aglomeraciones estudiadas. En los casos de estudio se seleccionaron los municipios que mayor relación funcional, poblacional y económica presentan en torno a las ciudades principales.

En el caso de Bogotá, (Figura 1), se analizaron los indicadores en conjunto con los municipios de Soacha, Funza, Madrid, Mosquera, Chía y Cajicá, dado los fenómenos de conurbación presentados y la intensidad de sus relaciones funcionales. Entre la ciudad principal y los municipios se estima una población total cercana a los 9.200.000 habitantes según las proyecciones realizadas por el Departamento Administrativo Nacional de Estadística [DANE], (2020). En la aglomeración, según los resultados del Censo del año 2018, el 99,2\% de la población reside en el área urbana aglomerada y conurbada. El PIB de la aglomeración representa cerca del 27,0\% del total de Colombia y está por encima de varios países de América Latina.

En el caso de Medellín, (Figura 1), se analizaron los indicadores en conjunto con los municipios pertenecientes al área metropolitana, es decir Bello, Copacabana, Caldas, Itagüí, La Estrella, Envigado y Sabaneta, los fenómenos de conurbación presentados y la intensidad de sus relaciones funcionales. Entre la ciudad principal y los municipios se estima una población total cercana a los 4.000 .000 habitantes según las proyecciones realizadas por el DANE (2020). En esta área metropolitana, según los resultados del Censo del año 2018, el 98,1\% de la población reside en el área urbana y su aporte al PIB nacional fue de cerca del 14,0\%.

Por último, en Cali, (Figura 1), se analizó la aglomeración en conjunto con Jamundí y Yumbo. Entre la ciudad principal y los municipios se estima una población total cercana a los 2.600 .000 habitantes según las proyecciones realizadas por el DANE (2020). Al igual que en los dos casos anteriores, aproximadamente el 98,4\% de la poblacion se concentra en el suelo urbano de los municipios. Es encesario resaltar que Cali es el principal centro urbano, económico e industrial del suroccidente del país y se consolidó como punto de intercambio económico a nivel nacional e internacional. Según cálculos de la administración de Santiago de Cali, su aporte al PIB nacional es de 9,7\%.

\subsection{Métodos}

La estimación de indicadores de segregación supone una serie de decisiones metodológicas relacionadas con la temporalidad, la escala de la información, la clasificación y las medidas estadísticas para poder estimar qué tan significativo es el fenómeno en un territorio (Rodríguez, 2013). En el caso de las ciudades de estudio, se ha identificado que la mejor fuente, por su metodología, variables y escala es el Censo Nacional de Población y Vivienda realizado por el Departamento Administrativo Nacional de Estadística (DANE). El Censo es una operación estadística que consiste en recoger, evaluar, analizar y difundir datos demográficos y sociales, relacionados con las personas, los hogares y las viviendas en Colombia (DANE, 2018).

En términos temporales, se cuenta con los resultados conciliados del censo realizado por el DANE en los años 2005 y 2018, los cuales son comparables en términos de variables de información y escala desagregación de los resultados. Esto permite tener resultados para dos momentos del siglo XXI, a partir de los cuales se puede dar cuenta de patrones de consolidación, transición o transformación de la segregación residencial en las ciudades de estudio. Si bien el óptimo sería contar con información longitudinal para todos los años de estudio, en el caso de las ciudades colombianas no es posible dada la ausencia de información, lo cual supone una limitación que no es posible superar en el marco de esta investigación.

Sin embargo, la propuesta metodológica supone un avance con respecto a los estudios realizados sobre segregación en Bogotá, Medellín y Cali, los cuales solo han tomado un año de referencia. La revisión de literatura arroja como resultado que principalmente se han tomado datos del censo del año 2005 y la información de estratos socioeconómicos ${ }^{4}$ disponible en el año de la investigación, pero no hay ninguna que compare diferentes mediciones en el tiempo (Mayorga, 2019).

Con respecto a la escala de la información, para los dos años de estudio, se obtuvieron resultados en una unidad mínima de manzana censal, lo cual permite la agrupación en otras unidades espaciales y la

\footnotetext{
4 Índice público en el que se evalúan los materiales de las viviendas y el entorno urbano, asignando un número de estrato del 1 al 6 a un grupo de viviendas que, por lo general, corresponde a una manzana (Secretaría Distrital de Planeación, 2007).
} 
estimación de indicadores de segregación. Una de las principales ventajas de contar con la información a mico escala es que se puede describir de mejor forma el entorno urbano de los grupos sociales y construir relaciones de vecindad o contigüidad espacial y estimar el grado en el que estos entornos urbanos difieren entre los distintos grupos sociales (Garroncho \& Campos, 2013). Lo anterior implica un avance metodológico en la medición de la segregación, dado que el fenómeno ha sido investigado principalmente en unidades político administrativas macro que no responden a la realidad territorial de las ciudades investigadas (Mayorga, 2019). En ese sentido, en la investigación se pasa de la generación de resultados a escala macro, para obtener una tendencia espacial a escala de manzana que permita estimar indicadores a escala zonal. Esto permite contrastar la segregación residencial en diferentes escalas.

La tercera decisión, se relaciona con la clasificación de los grupos sociales. Para ello, se recurre a la construcción propia de un Índice de Calidad de Vida Básico (ICVB) partir de métodos multivariados que permitan sintetizar variables consideradas clave en la medición del nivel socioeconómico de la población. Esto se hace teniendo en cuenta que los indicadores simples usados en otras investigaciones (nivel de estudios del jefe de hogar, por ejemplo) no permiten obtener otras dimensiones clave en la medición y, por otro lado, los indicadores de pobreza o calidad de vida se estiman para el total municipal y no para unidades espaciales más ajustadas (Mayorga, 2019).

\subsubsection{Estimación de Índice de Calidad de Vida Básico}

Las decisiones sobre la temporalidad, la escala y la clasificación de los grupos sociales, se manifiestan en la construcción de un Índice de Calidad de Vida Básico que permite clasificar a los grupos sociales según el nivel que tengan. Así mismo, este índice se construye para los dos años de estudio y a una escala micro, para evaluar los cambios espaciales que presenta.

Para la construcción del indicador propio, se seleccionaron variables disponibles y comparables en los dos censos. La selección de las variables a incluir en el indicador construido estuvo relacionada con las decisiones teóricas tomadas, así como con la disponibilidad de información disponible para consulta pública por el DANE. Como puede observarse, al igual que en otras investigaciones (Mayorga, 2017), se incluyeron las variables de vivienda y entorno, de dependencia económica y de logro educativo que evalúa la metodología de Necesidades Básicas Insatisfechas.

Las variables incluidas para la estimación del ICVB en el año 2005 y en el año 2018 son:

Tabla 1. Dimensiones e indicadores incluidos

\begin{tabular}{|c|c|}
\hline Dimensiones & Indicador/variable \\
\hline Hacinamiento no mitigable & Hogares por vivienda \\
\hline Tipología de vivienda & Tasa de hogares en viviendas según tipo \\
\hline Cobertura de servicios públicos & $\begin{array}{l}\text { Tasa de hogares sin servicio de acueducto } \\
\text { Tasa de hogares sin servicio de alcantarillado } \\
\text { Tasa de hogares sin servicio de energía }\end{array}$ \\
\hline Tamaño del hogar & Promedio de personas por hogar \\
\hline Actividad desarrollada por los integrantes del hogar & $\begin{array}{c}\text { Tasa de dependencia económica } \\
\text { Tasa de población mayor de } 17 \text { años sin empleo }\end{array}$ \\
\hline Grado educativo alcanzado por los integrantes del hogar & $\begin{array}{l}\text { Promedio de años de estudio alcanzado } \\
\text { Tasa de población mayor de } 17 \text { años sin educación }\end{array}$ \\
\hline No consumo de alimentos en la última semana & $\begin{array}{c}\text { Tasa de personas que no consumieron ninguna de las } 3 \text { comidas, } \\
\text { algún día de la última semana }\end{array}$ \\
\hline
\end{tabular}

Fuente: Mayorga, García y Hernández (2017).

Los indicadores, tanto en el año 2005 y 2018, fueron estandarizados para poder ser comparados entre sí. Posteriormente, se recurrió al análisis factorial por componentes principales para producir un índice compuesto con el peso de cada indicador en el ICVB. Se optó por un análisis de componentes principales teniendo en cuenta que el objetivo era reducir las dimensiones inicales a un conjunto menor de componentes que expliquen la varianza total observado. Esto, resulta más adecuado que otros métodos de extracción en el análisis factorial, en las que el objetivo principal es identificar las dimensiones que dan cuenta en mayor medida de la varianza común de las demás dimensiones (Mavrou, 2015). 
Se usó la técnica para generar agrupaciones de variables a partir de las correlaciones que presentan entre ellas. En ese sentido, la técnica permite encontrar grupos de variables que se pueden sintetizar en una sola medida común (Mayorga, García y Barrera, 2019).

Siguiendo las recomendaciones de la técnica hechas por, según Montoya (2007), Vallejo (2013), y Yong y Pierce (2013) se realizaron los siguientes pasos:

1. Elaboración de la Matriz de Correlaciones: se busca realizar un cálculo de una matriz capaz de expresar la variabilidad conjunta de las variables.

2. Extracción de los factores comunes.

3. La rotación de los factores iniciales: ayuda a clarificar la estructura subyacente a las variables y se pretende encontrar una estructura más simple y de más fácil interpretación.

4. Denominación a los factores encontrados.

Con los resultados del ICVB en los dos años, se hizo un análisis tendencial que partiera de la autocorrelación espacial de los resultados. A partir de la aplicación de un Índice Global de Moran, se confirmó si los datos presentaban una tendencia a la conformación de conglomerados en las ciudades según los resultados del ICVB.

Una vez identificada la autocorrelación espacial, del ICVB se procedió a generar un modelo interpolación que permitiera establecer la tendencia de distribución del ICVB y compararla en los dos años. Teniendo en cuenta que los datos se calcularon a escala de manzana, para dar cuenta de la tendencia espacial de los resultados y poder reconocer los patrones de conglomeración de los grupos se optó por realizar un modelo geoestadístico Kriging.

El modelo de interpolación Kriging, está basado en modelos estadísticos que incluyen la auto correlación, es decir, las relaciones estadísticas entre los puntos medidos. Gracias a esto, el modelo, no solo tienen la capacidad de producir una superficie de predicción, sino que también proporciona alguna medida de certeza o precisión de las predicciones. ${ }^{5}$

La fórmula general para ambos interpoladores se forma como una suma ponderada de los datos:

$$
Z\left(S_{O}\right)=\sum_{i=1}^{N} \lambda_{i} Z\left(S_{i}\right)
$$

Donde:

$\mathrm{Z}(\mathrm{si})=$ el valor medido en la ubicación i

$\lambda \mathrm{i}=$ una ponderación desconocida para el valor medido en la ubicación $\mathrm{i}$

s0 = la ubicación de la predicción

$\mathrm{N}$ = la cantidad de valores medidos

Los indicadores de segregación residencial se estimaron a partir de los resultados obtenidos en las manzanas censales de las ciudades de estudio. Al utilizar las manzanas como unidad mínima se obtienen 50.446 unidades espaciales para Bogotá, 20.571 para Medellín y 20.660 para Cali, lo cual asegura la suficiente varianza de los datos para obtener la distribución espacial de los grupos. En cada manzana se calculó el número de hogares según el ICVB fuera bajo (Menor a 0), medio (entre 0 y l) o alto (Mayor a 1), teniendo en cuenta la desviación de los datos.

Posteriormente con la clasificación de los hogares según la manzana en la que residen, se estimaron indicadores de segregación a dos escalas ${ }^{6}$ :

5. Para dar cuenta del fenómeno a escala macro, se utilizaron las localidades en Bogotá y las comunas en Cali y Medellín, de manera tal que se pudiera establecer la incidencia a una escala mayor de ciudad. Teniendo en cuenta que en las tres ciudades se analizaron los municipios vecinos como parte del continuo urbano, estos últimos se agruparon cada uno como localidad o comuna. Con esta escala, se estiman los indicadores de segregación en 26 unidades espaciales macro en Bogotá, 18 en Cali y 26 en Medellín.

5 El método Kriging presupone que la distancia o la dirección entre los puntos de muestra reflejan una correlación espacial que puede utilizarse para explicar la variación en la superficie. Se ponderan los valores medidos circundantes para calcular una predicción de una ubicación sin mediciones.

6 Es necesario acotar que los tamaños de las localidades, las comunas y los barrios difieren entre las tres ciudades. 
6. Se estimaron los indicadores a escala barrial en cada una de las ciudades, para establecer qué tanta segregación hay en los entornos más próximos a la vivienda de los hogares. Esto permitió la estimación de los indicadores de segregación en 704 unidades espaciales micro de Bogotá, 420 de Medellín y 440 de Cali.

Las escalas de análisis se pueden observar a continuación:

Figura 2. Escalas de análisis en Bogotá, Medellín y Cali

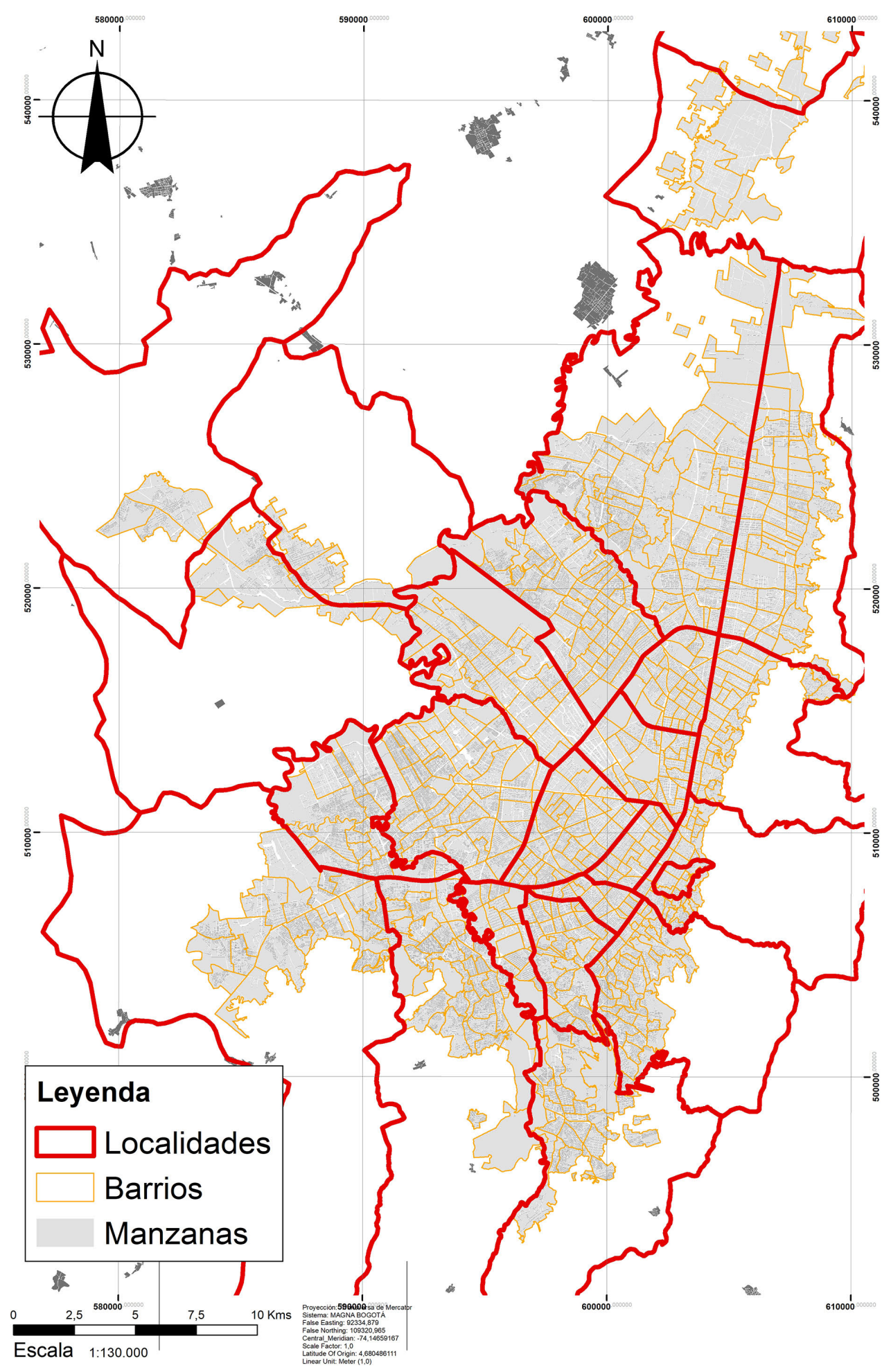




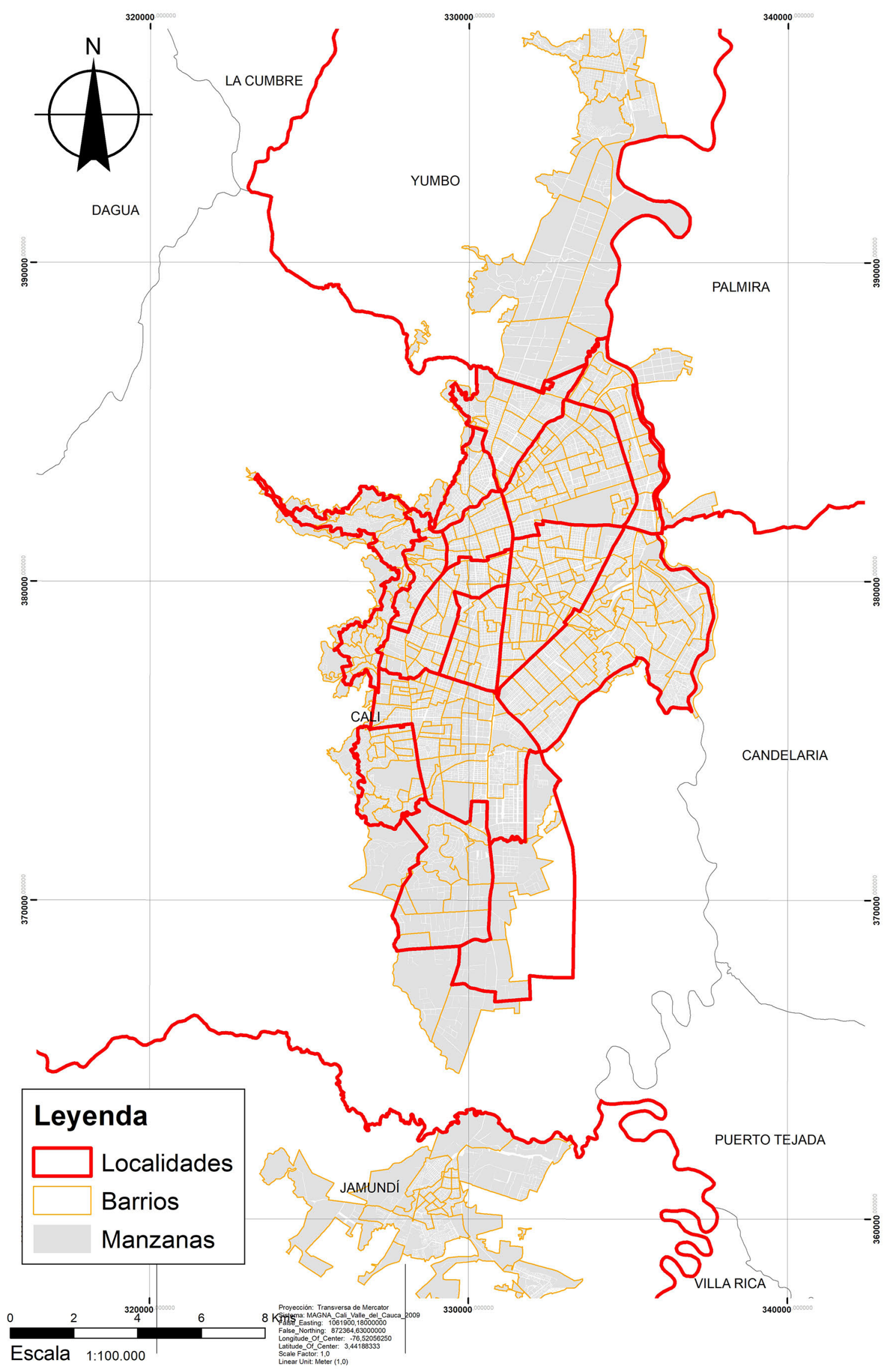




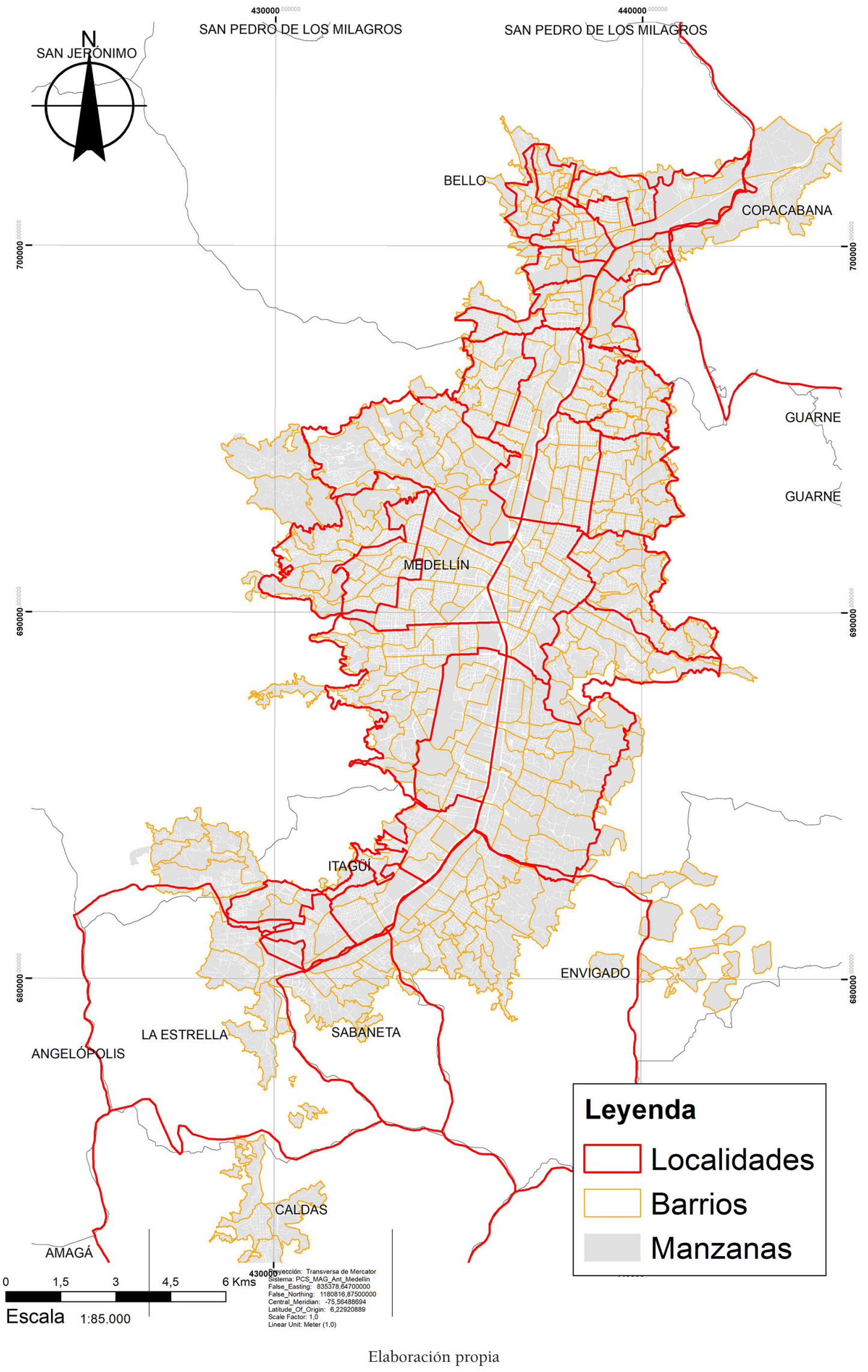


A estas dos escalas se aplicaron indicadores de segregación que incluyeran una variable espacial, de tal forma se optó por calcular el Índice de segregación ajustado por la forma, el índice de disimilitud ajustado por la forma y el índice de interacción. Los dos primeros corresponden a índices de desigualdad, mientras que el último a índices de exposición.

El indicador de Segregación ajustado por la longitud de la frontera y la relación perímetro / superficie tiene la siguiente nomenclatura:

$$
\text { IS (S) }=I S-\frac{1}{2} \sum_{i=1}^{n} \sum_{j=1}^{n} W_{i j}\left|Z_{i}-Z_{j}\right| * \frac{\frac{1}{2}\left[\left(\frac{p_{i}}{a_{i}}\right)+\left(\frac{p_{j}}{a_{j}}\right)\right]}{\max \left(\frac{p_{i}}{a_{i}}\right)}
$$

Donde:

$W_{i j}=$ elemento de la matriz de distancias, $Z_{i}=$ proporción del grupo $X$ en la unidad $i$

$Z_{j}=$ proporción del grupo $X$ en la unidad $j, p_{i}=$ perimetro de la unidad $i$

$p_{j}=$ perimetro de la unidad $j, a_{i}=$ superficie de la unidad $i$

$a_{j}=$ superficie de la unidad $j$

Los resultados del indicador varían entre 0 y 1 Se considera que hay una segregación alta de un grupo, cuando el valor está por encima de 0,7 (Martori, Hoberg y Surinach, 2006).

El indicador de Disimilitud ajustado por la longitud de la frontera y la relación perímetro / superficie tiene la siguiente nomenclatura:

$$
\mathrm{D}(\mathrm{S})=D-\frac{1}{2} \sum_{i=1}^{n} \sum_{j=1}^{n} W_{i j}\left|Z_{i}-Y_{j}\right| * \frac{\frac{1}{2}\left[\left(\frac{p_{i}}{a_{i}}\right)+\left(\frac{p_{j}}{a_{j}}\right)\right]}{\max \left(\frac{p_{i}}{a_{i}}\right)}
$$

Donde:

$W_{i j}=$ elemento de la matriz de distancias, $Z_{i}=$ proporción del grupo $X$ en la unidad $i$

$Y_{j}=$ proporción del grupo $Y$ en la unidad $j, p_{i}=$ perimetro de la unidad $i$

$p_{j}=$ perimetro de la unidad $j, a_{i}=$ superficie de la unidad $i$

$a_{j}=$ superficie de la unidad $j$

Los resultados del indicador varían entre 0 y 1 Se considera que hay una disimilitud alta de un grupo, cuando el valor está por encima de 0,7 (Martori et al., 2006).

El indicador de Interacción tiene la siguiente nomenclatura:

$$
x P y=\sum_{i=1}^{n}\left(\frac{x_{i}}{X}\right)\left(\frac{y_{i}}{t_{i}}\right)
$$

Donde:

$x_{i}=$ población del grupo $X$ en la unidad $i, X=$ población del grupo $X$ en la ciudad

$y_{i}=$ población del grupo $Y$ en la unidad $i, t_{i}=$ población total de la unidad $i$

El índice varía entre 0 y 1 y se considera que hay una interacción alta, cuando el valor está por encima de 0,7 (Martori et al., 2006).

\section{Resultados}

Al realizar el análisis factorial en las tres ciudades, se obtuvo como resultado que las once variables incluidas se resumen a tres factores que logran explicar el 73,2\% (2005) y el 74,1\% (2018) de la varianza en el caso de Bogotá, 74,5\% (2005) y 71\% (2018) en el caso de Medellín y 69,6\% (Año 2005) y 73,6\% (Año, 2018) en el caso de Cali, lo cual le da validez al modelo estadístico en las tres ciudades.

En todos los casos se obtuvo un KMO superior a 0,7 y se puede considerar como validada la prueba y las 11 variables se adecuan de manera aceptable a los factores expresados en el modelo. Igualmente se comparó la prueba de esfericidad de Bartlett, que con un de, valida el modelo en las tres ciudades estu- 
diadas. Con respecto a las comunalidades, se identifica que en las tres ciudades hay una extracción de más del 50,0\% de las variables utilizadas, lo cual valida nuevamente el modelo.

Mediante una rotación varimax de las variables, en las tres ciudades se obtuvo que los componentes principales del factor 1 son el ayuno, el promedio de años de educación de la población, el porcentaje de población sin educación y el porcentaje de población desempleada. Teniendo en cuenta que las variables están asociadas a las características de los individuos y su inserción en la estructura económica, se denomina factor socioeconómico (Mayorga et al., 2017).

El factor 2 tiene como componentes principales son el porcentaje de viviendas con energía eléctrica, el porcentaje de viviendas con servicio de acueducto y el porcentaje de viviendas con servicio de alcantarillado. Ya que las variables están asociadas a las características de la prestación de servicios públicos en la vivienda, se denomina factor de servicios públicos en la vivienda (Mayorga et al., 2017).

Por último, el factor tiene como componentes principales son el número de hogares por vivienda, el número de personas por hogar y el indicador de dependencia económica. Estás variables se asocian a la población con respecto a su estructura etárea, la composición del hogar y la distribución de viviendas en los hogares, por lo cual se denomina factor de composición demográfica (Mayorga et al., 2017).

Por último, para obtener un índice compuesto en las tres ciudades, se calculó un promedio simple entre los tres factores, atribuyendo a todos los mismos pesos. En adelante, este índice se denomina en adelante ICV básico.

$$
I C V=\frac{F 1+F 2+F 3}{3}
$$

F1: Características socioeconómicas. F2: Servicios públicos en la vivienda. F3: Composición demográfica.

En el caso de la aglomeración de Bogotá en el año 2005, se obtiene como resultado que el 37\% de la población tiene un ICVB clasificado como bajo, el $47 \%$ se encuentra clasificado con un índice medio y el 15\% con un índice alto. Para el año 2018, se identifica una transformación en el peso porcentual de cada grupo, ya que el grupo clasificado con ICVB bajo pasa a pesar el 31\% (disminución del 6\%), mientras que el grupo con ICVB medio crece un 3\% para pasar a pesar el 50\% y el grupo con ICVB alto crece un $4 \%$ y queda en el 19\%. En términos brutos, se da un incremento en los grupos medios y altos, mientras que se da una leve reducción de los grupos bajos.

Figura 3. Distribución porcentual de grupos por ICVB en Bogotá años 2005 y 2018

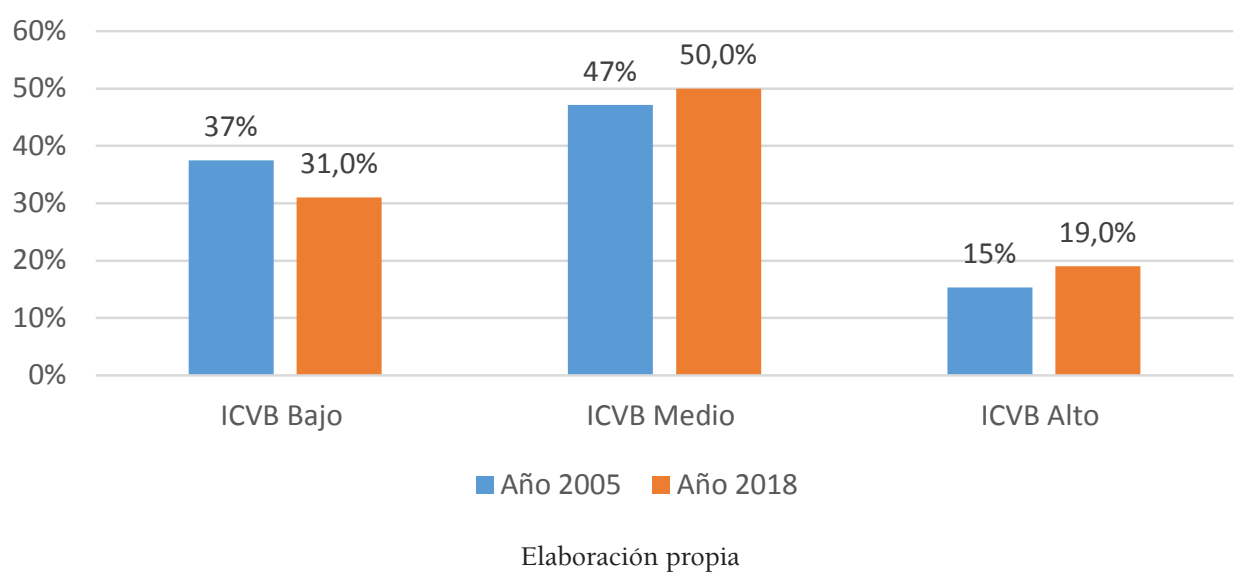

Al aplicar el índice de Moran, con el ICVB para los resultados obtenidos en el año 2005, con un P valor menor a un nivel de significancia de 0,05, se acepta la hipótesis de que los valores presentan auto correlación espacial y se agrupan en conglomerados. Al realizar la interpolación para establecer la tendencia espacial, se puede observar que en el año 2005 hay una concentración de los grupos altos en una cuña de alta renta que va del centro hacia el norte de la ciudad, por el borde oriental, con una pronunciación hacia 
el occidente en su inicio al sur. Por el contrario, se identifica una concentración de grupos con ICVB bajo hacia el sur y occidente de la ciudad, con una tendencia marcada hacia los municipios colindantes con la misma tendencia. Los grupos medios se establecen como un colchón intermedio entre la distribución de grupos bajos y altos.

Para el año 2018, las pruebas estadísticas autocorrelación espacial indican que se trata de un fenómeno que mantiene la tendencia de conformación de conglomerados. La tendencia espacial del año 2005 se refuerza, con el evidente crecimiento de la distribución de grupos con ICVB alto al norte la ciudad, en los municipios de Chía y Cajicá y consolidación de una nueva cuña hacia el occidente de la ciudad por el eje de la Avenida El Dorado (vía al aeropuerto internacional). Así mismo se evidencia la aparición de lo que Janoschka (2002) llama islas de grupos socioeconómicos altos al noroccidente de la ciudad. Con respecto a los grupos con ICVB bajo se mantiene su distribución al sur y al occidente en las periferias de la ciudad. Si bien se puede afirmar que el grupo disminuyó en términos brutos y porcentuales, su distribución espacial es reforzada en una tendencia de concentración al sur de la ciudad, con la presencia de algunos grupos medios de manera intermitente en algunas islas.

Figura 4. Tendencia del ICVB en Bogotá 2005 y 2018

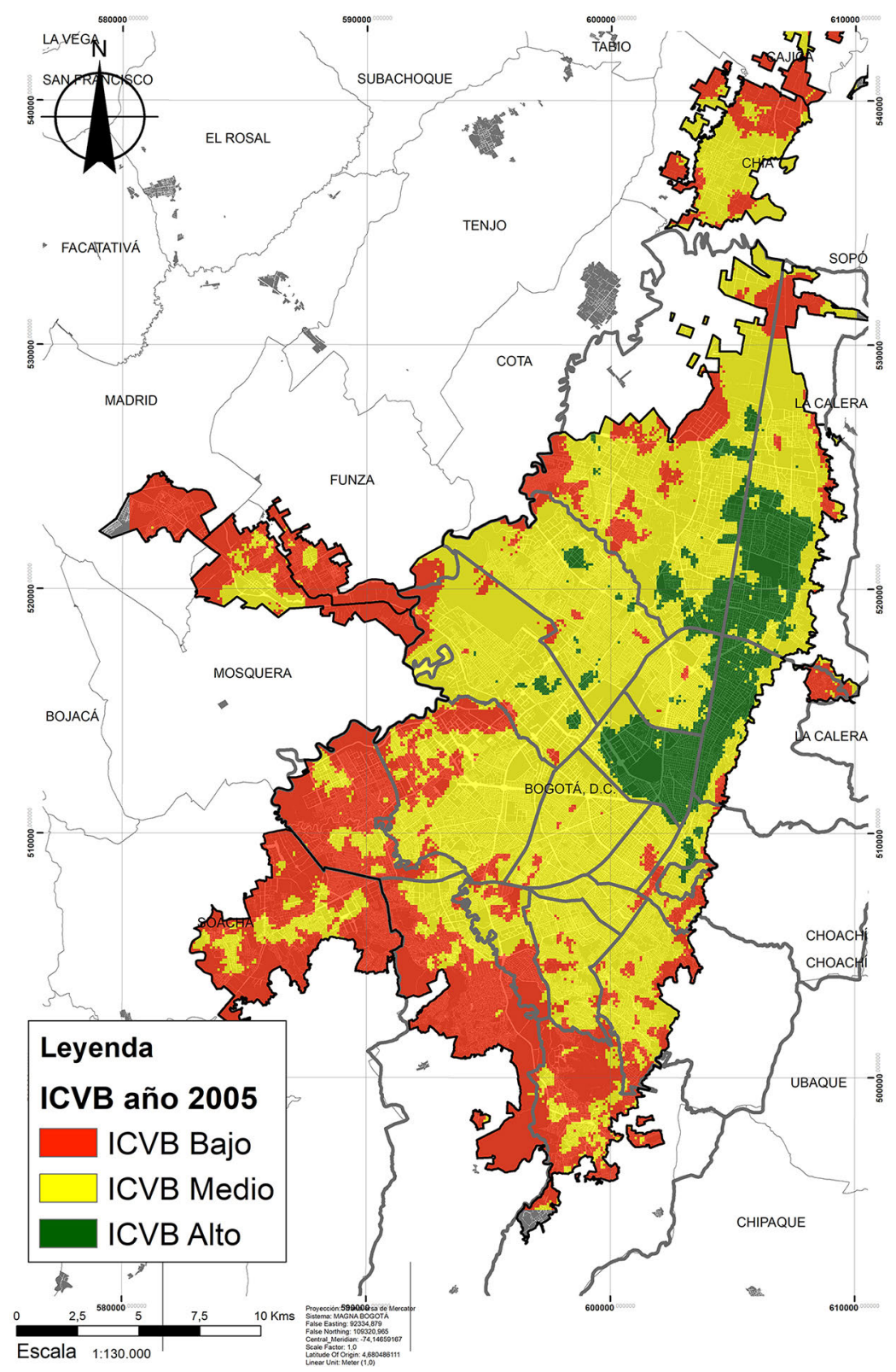




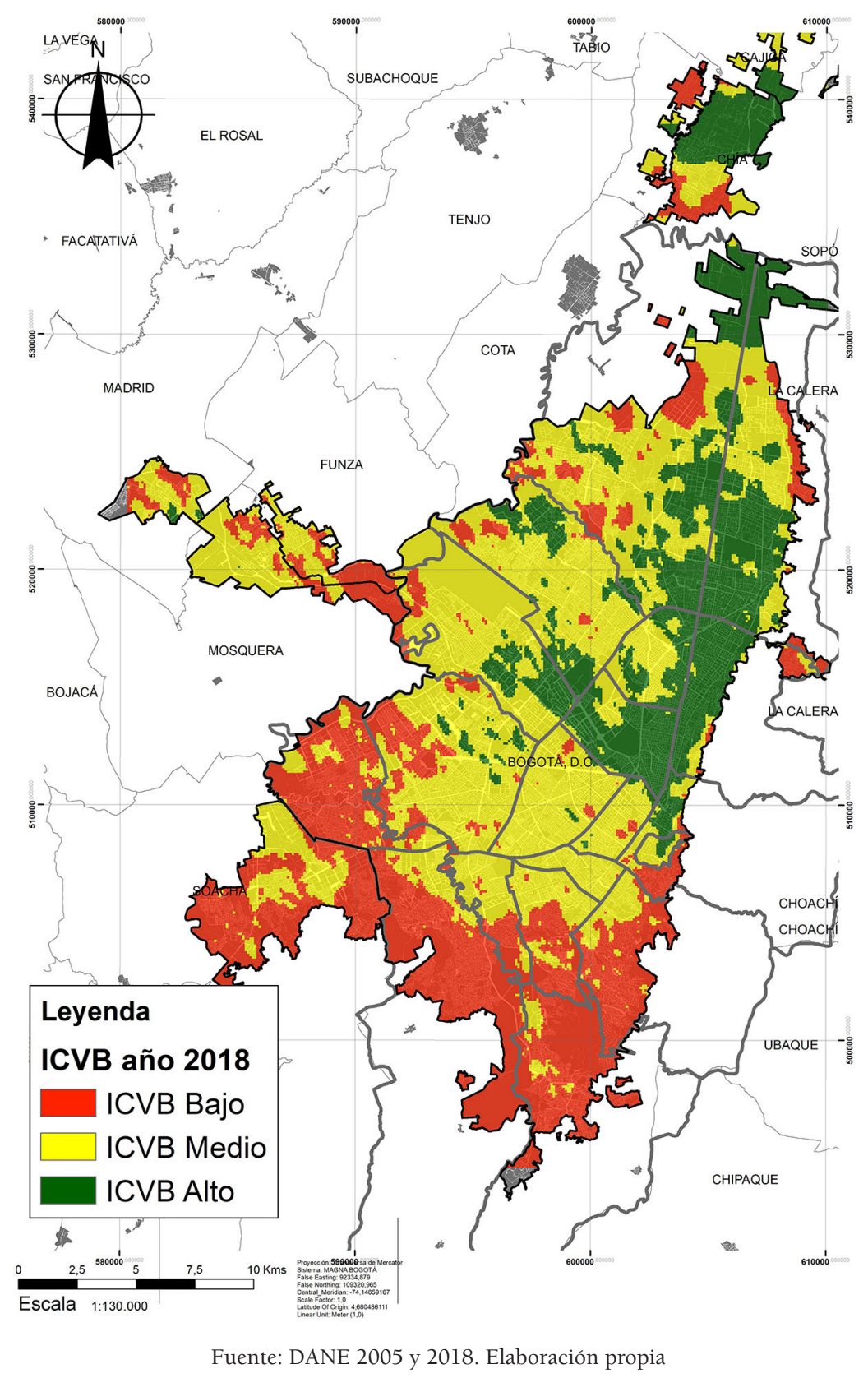

En el caso de la aglomeración de Medellín en el año 2005, se obtiene como resultado que el 37,5\% de la población tiene un ICVB clasificado como bajo, el 48,2\% se encuentra clasificado con un índice medio y el 14\% con un índice alto. Para el año 2018, se identifica una transformación en el peso porcentual de cada grupo, ya que el grupo clasificado con ICVB bajo pasa a pesar el 26,7\% (disminución del 11\%), mientras que el grupo con ICVB medio crece un $10 \%$ para pasar a pesar el $58 \%$ y el grupo con ICVB alto crece un 2,0\% y queda en el $16 \mathrm{~m} 2 \%$. Al igual que en Bogotá, en términos brutos, se da un incremento en los grupos medios y altos, mientras que se da una leve reducción de los grupos bajos.

Al aplicar el índice de Moran, con el ICVB para los resultados obtenidos en el año 2005, con un P valor menor a un nivel de significancia de 0,05, se acepta la hipótesis de que los valores presentan auto correlación espacial y se agrupan en conglomerados. Al realizar la interpolación para establecer la tendencia espacial, se puede observar que en el año 2005 hay una concentración de los grupos altos que difiere de los modelos de diferenciación de la ciudad latinoamericana y en especial de la tendencia presentada en Bogotá. En el caso de Medellín se identifica una distribución de grupos con ICVB alto hacia el sur oriente de la ciudad, con tendencia hacia el municipio de Envigado. Así mismo, se identifican unas islas 
en el centro y occidente de la aglomeración. Los grupos bajos se concentran en las partes altas del oriente y el occidente en el norte de la ciudad, con una tendencia hacia el municipio de Bello y algunos conglomerados menores hacia el valle de la ciudad, en inmediaciones del Río Medellín. Al igual que en Bogotá, el grupo con ICVB medio se establece como un colchón intermedio entre la distribución del grupo con ICVB bajo y alto, sin presentar una tendencia espacial clara.

Figura 5. Distribución porcentual de grupos por ICVB en Medellín años 2005 y 2018

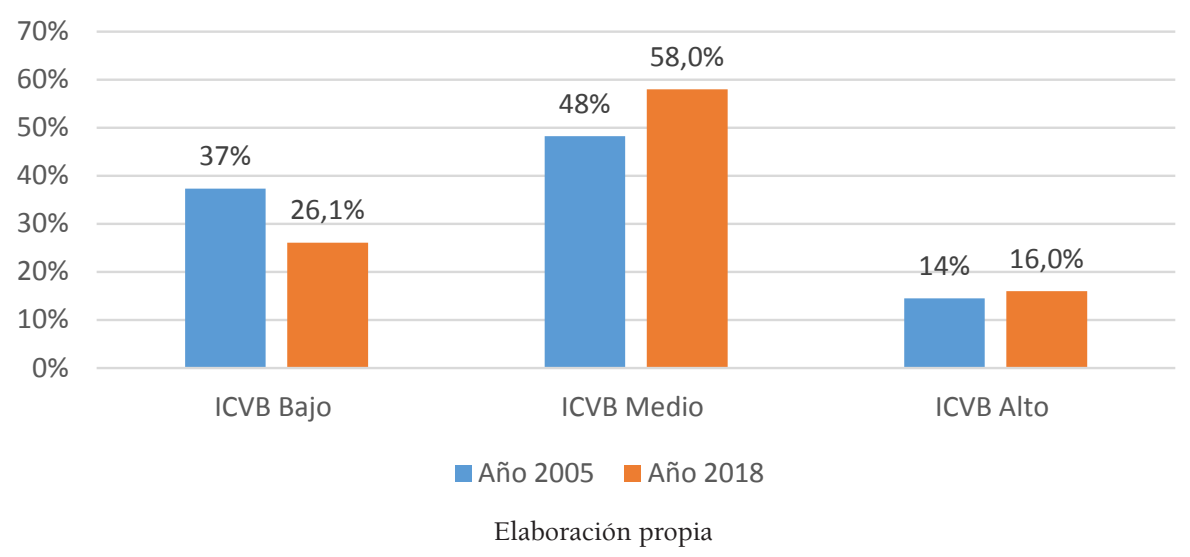

Figura 6. Tendencia del ICVB en Medellín 2005 y 2018

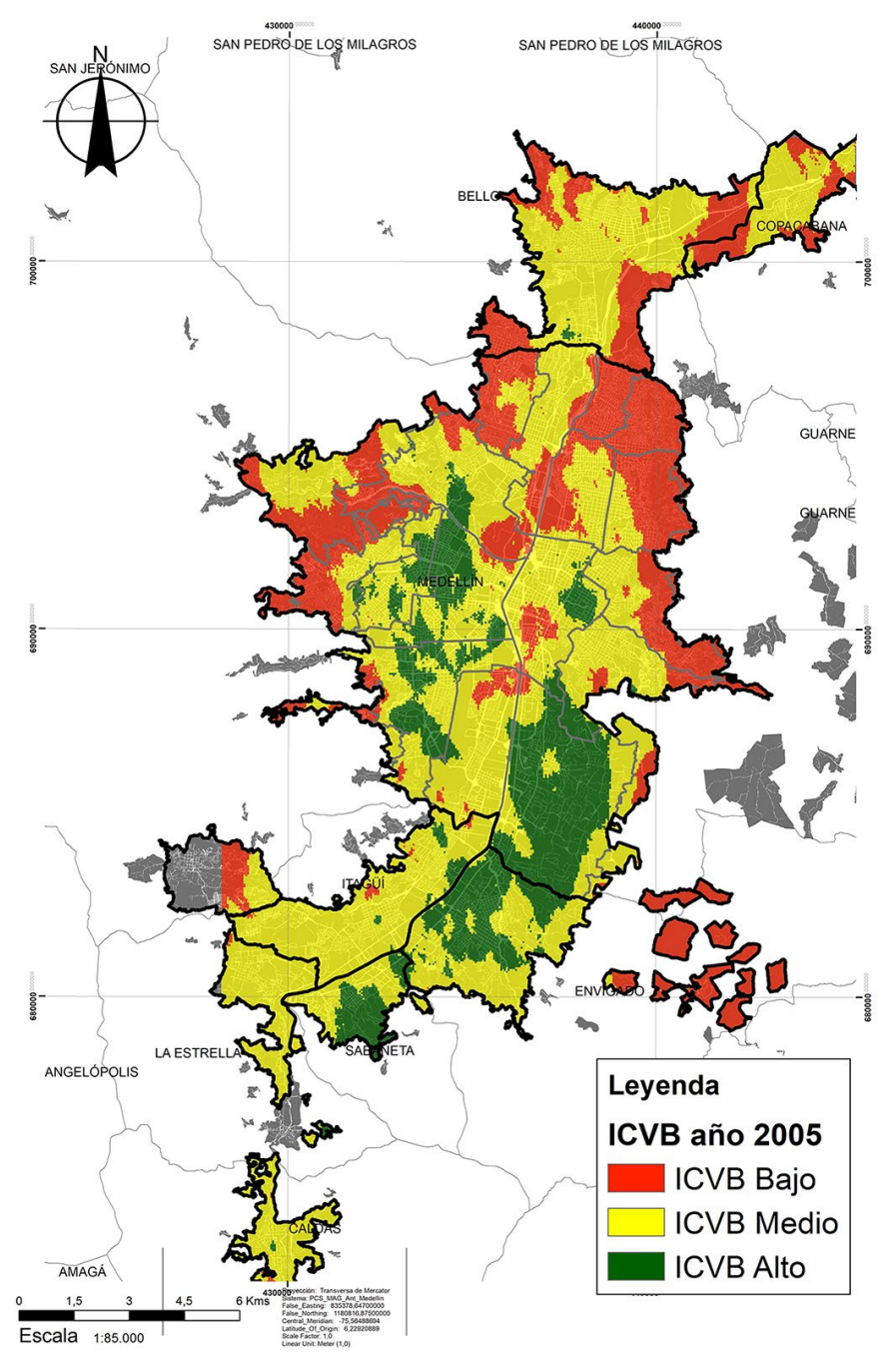




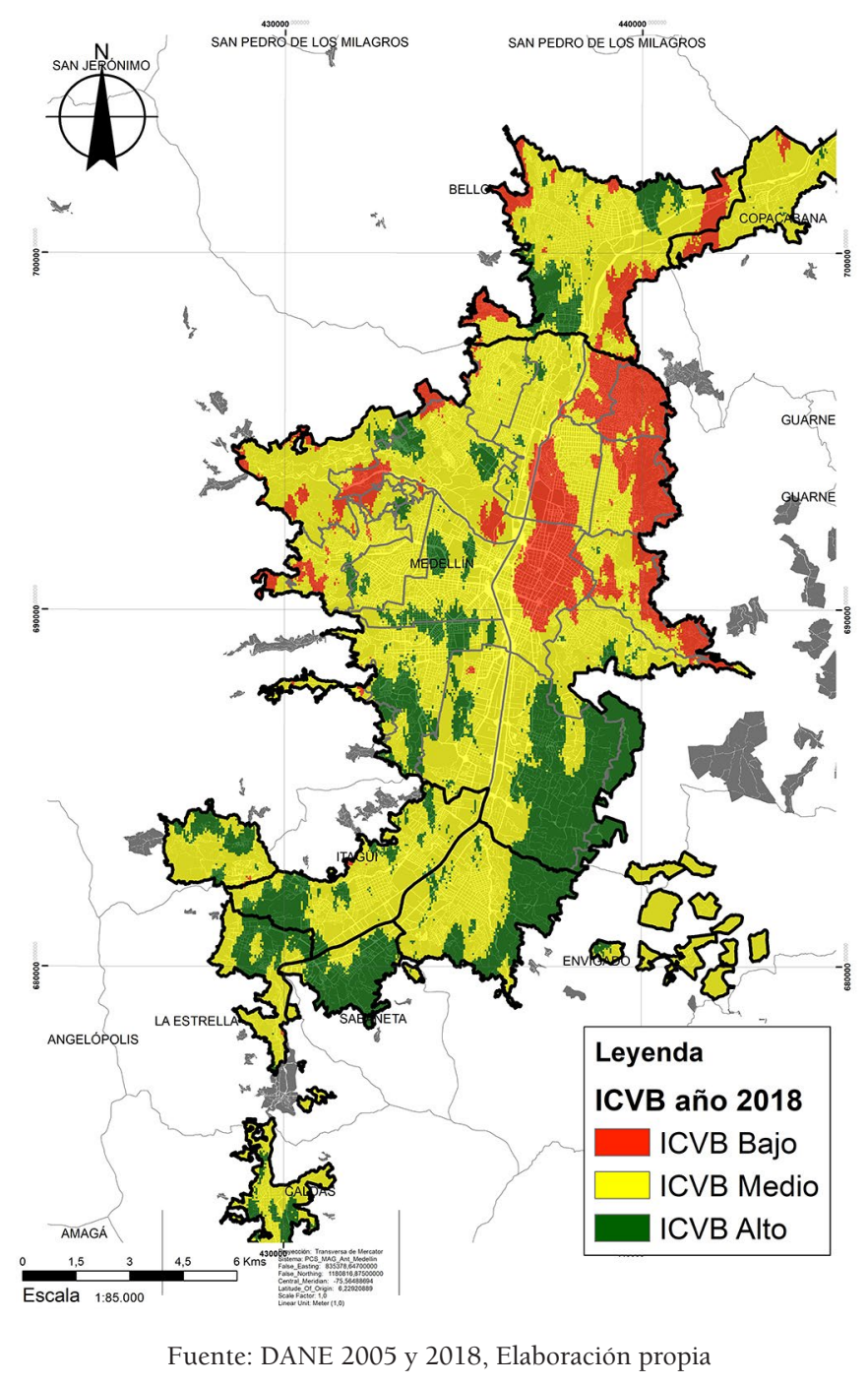

Para el año 2018, las pruebas estadísticas autocorrelación espacial indican que se trata de un fenómeno que mantiene la tendencia de conformación de conglomerados, aunque con algunas transformaciones. En el año 2018 se hace más marcada la presencia del grupo con ICVB alto hacia el oriente y el sur en dirección al municipio de Envigado. Sin embargo, aparecen unas islas de grupos altos en el norte y el occidente de la ciudad que generan una distribución que permite mayor interacción espacial con otros grupos. Con respecto a los grupos con ICVB bajo, se identifica su disminución y su concentración principalmente hacia la parte alta del oriente de la ciudad, con la consolidación de un conglomerado en torno al río Medellín en la parte baja. Resalta la disminución en el occidente de la ciudad.

En el caso de la aglomeración de Cali en el año 2005, el 47,3\% de la población tiene un ICVB clasificado como bajo, el 35,2\% se encuentra clasificado con un índice medio y el 18,6\% con un índice alto. Para el año 2018, se identifica una transformación en el peso porcentual de cada grupo, ya que el grupo clasificado con ICVB bajo pasa a pesar el 36\% (disminución del 12\%), mientras que el grupo con ICVB medio crece un $6,4 \%$ para pasar a pesar el $41 \%$ y el grupo con ICVB alto crece un $4,2 \%$ y queda en el $22,0 \%$. Al igual que en Bogotá y Medellín, en términos brutos, se da un incremento en los grupos medios y altos, mientras que se da una leve reducción de los grupos bajos.

Al aplicar el índice de Moran, con el ICVB para los resultados obtenidos en el año 2005, con un P valor menor a un nivel de significancia de 0,05, se acepta la hipótesis de que los valores presentan auto correlación espacial y se agrupan en conglomerados. Al realizar la interpolación para establecer la tendencia espacial, se puede observar que en el año 2005 hay una concentración de los grupos altos en un eje de alta renta que va del norte hacia el sur de la ciudad, por el borde occidental con dirección al municipio 
de Jamundí. Se identifica una concentración de grupos con ICVB bajo hacia el oriente de la ciudad en el denominado Distrito de Aguablanca y hacia las partes altas de la ciudad en el oriente de esta. Destaca igualmente la presencia de un conglomerado de ICVB bajo en el municipio de Yumbo, al norte de la aglomeración. Al igual que en Bogotá y Medellín, el grupo con ICVB medio se establece como un colchón intermedio entre la distribución del grupo con ICVB bajo y alto, sin presentar una tendencia espacial clara.

Figura 7. Distribución porcentual de grupos por ICVB en Cali años 2005 y 2018

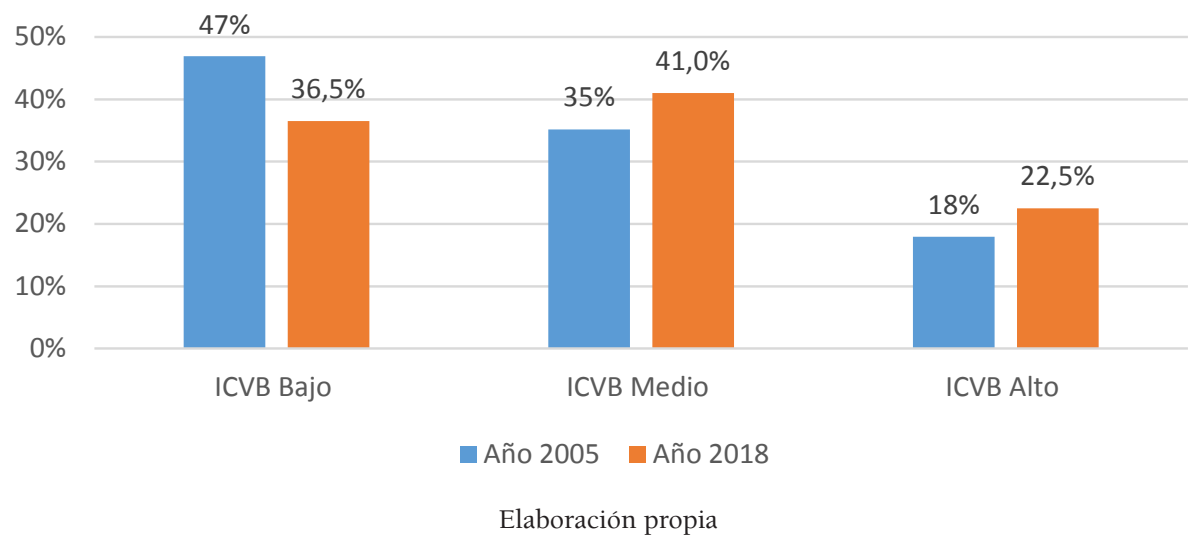

Figura 8. Tendencia del ICVB en Cali 2005 y 2018

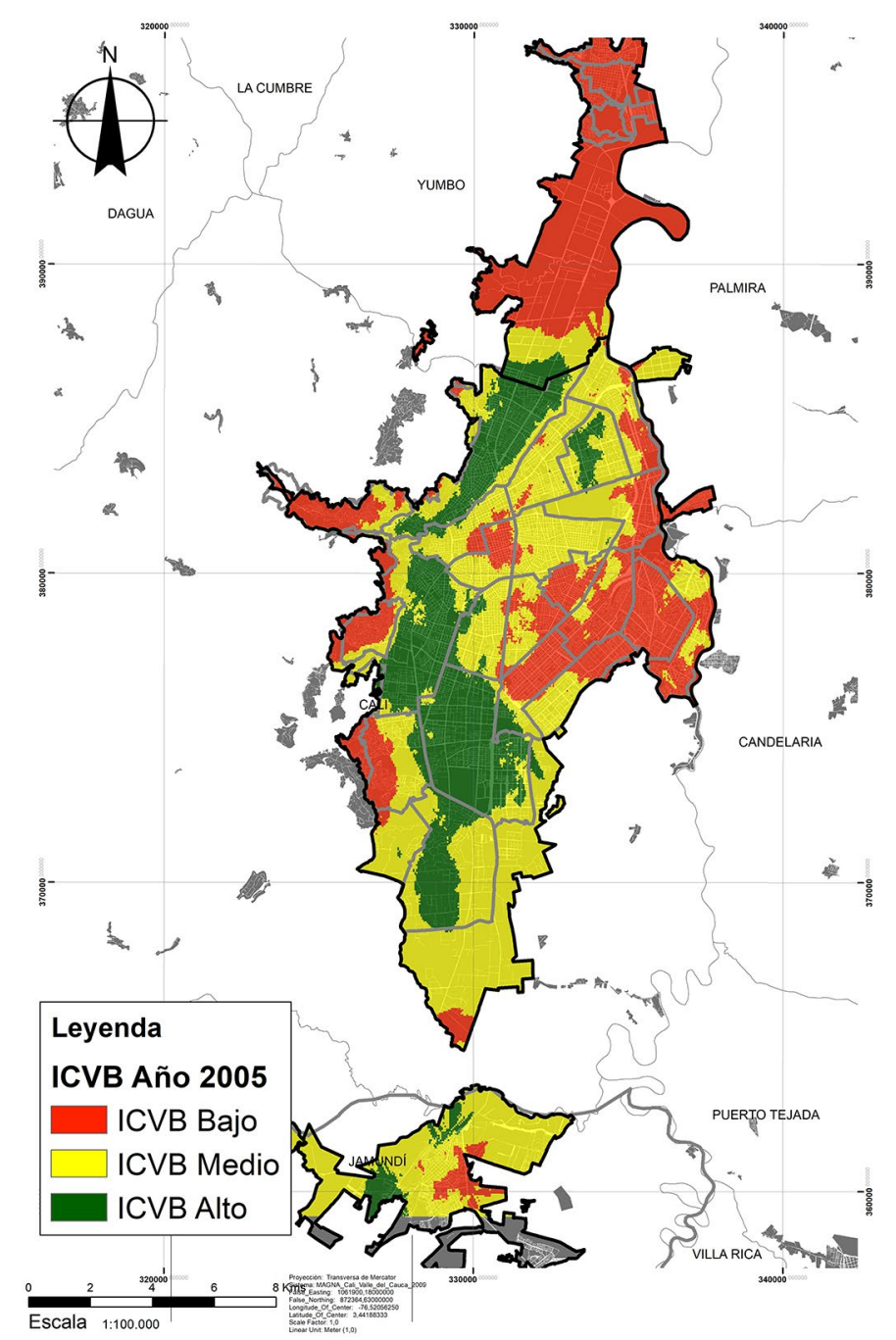




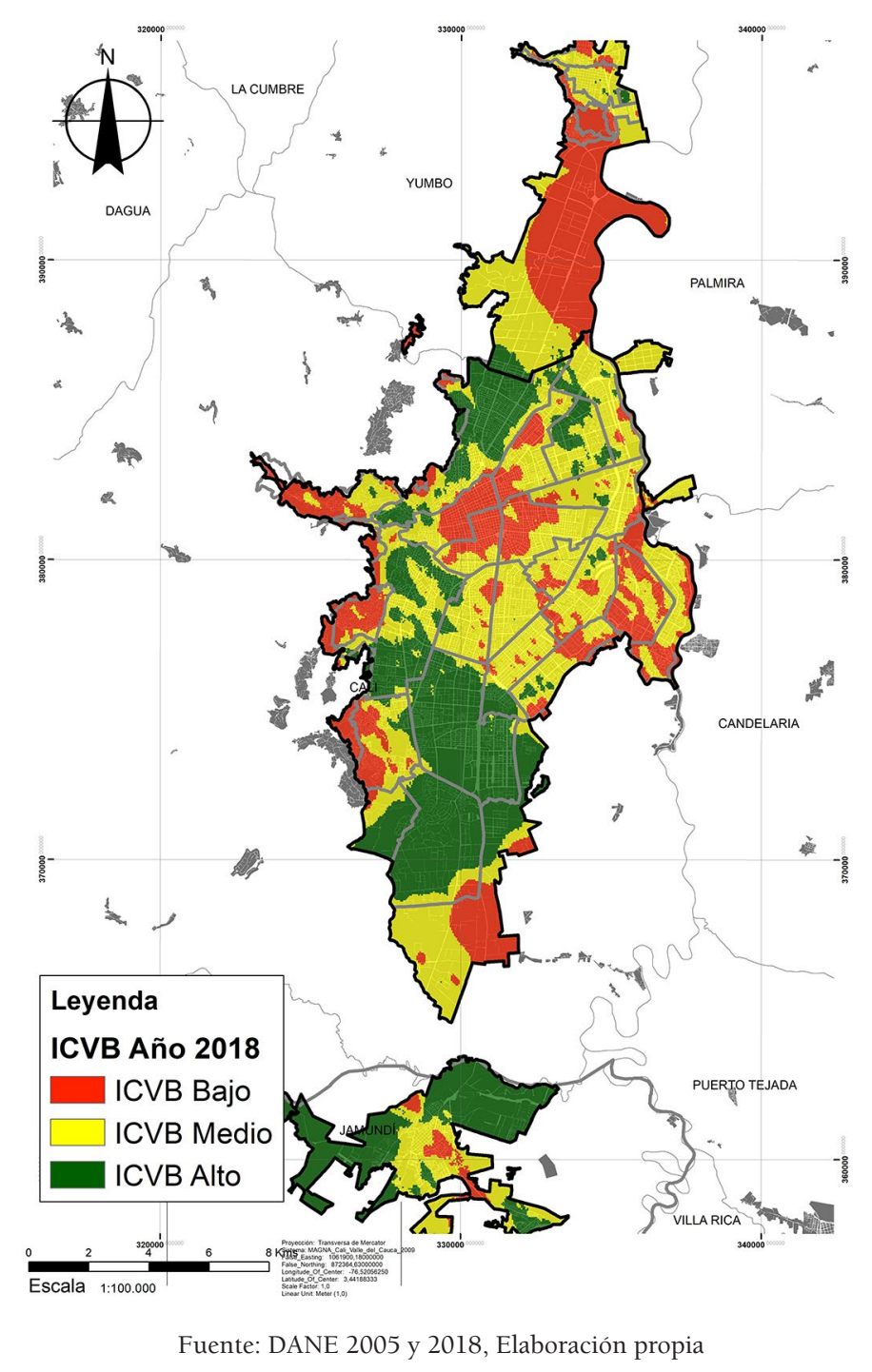

Para el año 2018, las pruebas estadísticas de autocorrelación espacial indican que se trata de un fenómeno que mantiene la tendencia de conformación de conglomerados. La tendencia espacial de conformación de conglomerados del grupo con ICVB alto, se consolida hacia el sur de la ciudad en la zona de Pance y el municipio de Jamundí. Con respecto a los grupos con ICVB bajo se mantiene su distribución al occidente y al oriente de la ciudad. Sin embargo, en el oriente de la ciudad se da una mayor relación con el grupo de ICVB medio, el cual es el que tiene un mayor crecimiento en términos brutos y porcentuales en la ciudad.

Como se aclara en la metodología, para calcular los indicadores de segregación se estimó la cantidad de habitantes según los rangos de ICVB a dos escalas: sector y localidades o comunas.

En el caso de Bogotá el Indicador de Segregación para el año 2005 a escala de sector muestra que la segregación más alta es la de los grupos con ICVB alto $(0,66)$, los cuales presentan una mayor tendencia a la conformación de conglomerados. La segregación del grupo con ICVB bajo puede ser considerado medio- alta, con un valor de 0,53. Sin embargo, este mismo indicador para el año 2005 tiende a ser significativamente inferior a escala de localidad, lo cual indica que hay una mayor incidencia de la segregación a escala de sector que a una escala mayor de ciudad, en la que los grupos están más integrados.

En el caso de Bogotá, el Indicador de Segregación para el año 2018 se incrementa en ambas escalas. En la escala menor, el grupo con ICVB alto sigue siendo el que mayor segregación presenta, pasando a 0,68, mientras el grupo con ICVB bajo se incrementa a 0,56. En la escala mayor (localidades), el único grupo que incrementa la segregación es el de ICVB bajo, en los otros dos grupos se mantiene el mismo valor que en el año 2005. 
Tabla 2. Indicador de Segregación en Bogotá por sector y localidad, años 2005 y 2018

\begin{tabular}{|l|c|c|c|c|}
\hline \multirow{2}{*}{ GRUPO } & \multicolumn{2}{|c|}{ Sector } & \multicolumn{2}{c|}{ Localidad } \\
\cline { 2 - 5 } & IS(s) 2005 & IS(s) 2018 & IS(s) 2005 & IS(s) 2018 \\
\hline ICVB Bajo & 0,53 & 0,56 & 0,32 & 0,38 \\
\hline ICVB Medio & 0,41 & 0,42 & 0,21 & 0,21 \\
\hline ICVB Alto & 0,66 & 0,68 & 0,46 & 0,46 \\
\hline
\end{tabular}

Elaboración propia

El Indicador de disimilitud ajustado por la longitud de la frontera y la relación perímetro / superficie permite concluir que en Bogotá la mayor disimilitud se presenta entre el grupo con ICVB bajo e ICVB alto, la cual se incrementa en la escala de sector entre los años 2005 y 2018, al pasar de 0,77 a 0,87 . Esta disimilitud es más baja a escala local, pero igualmente es la más significativa entre los grupos e igualmente se incrementa de 0,56 a 0,58 en los dos momentos analizados.

Tabla 3. Indicador de Disimilitud en Bogotá por sector y localidad, años 2005 y 2018

\begin{tabular}{|l|r|r|r|r|}
\hline \multirow{2}{*}{ GRUPOS } & \multicolumn{2}{|c|}{ Sector } & \multicolumn{2}{c|}{ Localidad } \\
\cline { 2 - 5 } & $(\mathrm{D}(\mathrm{s})) \mathbf{2 0 0 5}$ & $(\mathrm{D}(\mathrm{s})) \mathbf{2 0 1 8}$ & $(\mathrm{D}(\mathrm{s})) \mathbf{2 0 0 5}$ & $(\mathrm{D}(\mathrm{s})) \mathbf{2 0 1 8}$ \\
\hline ICVB Bajo - ICVB Medio & 0,47 & 0,51 & 0,27 & 0,28 \\
\hline ICVB Bajo - ICVB Alto & 0,77 & 0,87 & 0,56 & 0,58 \\
\hline ICVB Medio - ICVB Alto & 0,60 & 0,64 & 0,41 & 0,40 \\
\hline
\end{tabular}

Elaboración propia

Por último, teniendo en cuenta la distribución espacial de los grupos, se estima la probabilidad de interacción entre ellos. En el año 2005, se identifica que la probabilidad de interacción entre los grupos es baja en todos los casos, tanto a escala de sector como escala de localidad. Sin embargo, resalta que la probabilidad de interacción del grupo con ICVB bajo y el grupo con ICVB alto, está por debajo del 0,1 a escala de sector y solo llega a 0,15 a escala local, lo cual permite afirmar que hay una alta diferencia en la distribución de estos dos grupos en el espacio urbano de Bogotá y sus municipios vecinos. Este indicador se incrementa para el año 2018, ya que baja a 0,04 a escala de sector y a 0,13 a escala local, consolidando la tendencia de separación de los grupos.

Tabla 4. Indicador de Interacción en Bogotá por sector y localidad, años 2005 y 2018

\begin{tabular}{|l|r|r|r|r|}
\hline \multirow{2}{*}{ GRUPOS } & \multicolumn{2}{|c|}{ Sector } & \multicolumn{2}{c|}{ Localidad } \\
\cline { 2 - 5 } & (xPy)2005 & (xPy)2018 & (xPy)2005 & (xPy)2018 \\
\hline ICVB Bajo - ICVB Medio & 0,32 & 0,30 & 0,39 & 0,35 \\
\hline ICVB Bajo - ICVB Alto & 0,07 & 0,04 & 0,15 & 0,13 \\
\hline ICVB Medio - ICVB Alto & 0,24 & 0,23 & 0,28 & 0,21 \\
\hline
\end{tabular}

Elaboración propia

En Medellín los indicadores estimados demuestran que la incidencia de la segregación es sensiblemente inferior a la de Bogotá, y al contrario de lo sucedido en la capital del país, los indicadores disminuyeron en el periodo 1993-2018 tienden a disminuir para el grupo con ICVB alto.

El Indicador de Segregación para el año 2005 a escala de sector muestra que la segregación más alta es la de los grupos con ICVB alto $(0,69)$, los cuales presentan una mayor tendencia a la conformación de conglomerados. La segregación del grupo con ICVB bajo puede ser considerado media, con un valor de 0,49 . A una escala mayor, estos indicadores son más bajos, lo cual indica que hay una mayor incidencia de la segregación a escala de sector que a una escala mayor de ciudad.

Es necesario resaltar que para el año 2018 los indicadores de segregación del grupo con ICVB alto disminuyen tanto a escala de sector como a escala de comuna, pasando a 0,49 y 0,37 respectivamente, lo cual lleva a la conclusión que se dio una distribución más extendida de este grupo en el territorio. En los 
grupos con ICVB Bajo y Medio no se presentan cambios significativos en los indicadores de segregación, si bien se resalta que a escala local tienden a estar por debajo de 0,5 , lo cual quiere decir que se distribuyen de manera más integrada entre sí.

Tabla 5. Indicador de Segregación en Medellín por sector y comunas, años 2005 y 2018

\begin{tabular}{|l|c|c|c|c|}
\hline \multirow{2}{*}{ GRUPO } & \multicolumn{2}{|c|}{ Sector } & \multicolumn{2}{c|}{ Comuna } \\
\cline { 2 - 5 } & IS(s) 2005 & IS(s) 2018 & IS(s) 2005 & IS(s) 2018 \\
\hline ICVB Bajo & 0,49 & 0,52 & 0,41 & 0,43 \\
\hline ICVB Medio & 0,50 & 0,41 & 0,35 & 0,32 \\
\hline ICVB Alto & 0,69 & 0,49 & 0,59 & 0,37 \\
\hline
\end{tabular}

Elaboración propia

El Indicador de disimilitud ajustado por la longitud de la frontera y la relación perímetro / superficie en el caso de Medellín muestra que la mayor disimilitud se presenta entre el grupo con ICVB bajo e ICVB alto. Sin embargo, esta disimilitud disminuye en el periodo de análisis de 0,82 a 0,71 en la escala sectorial y de 0,67 a 0,58 en la escala de comuna. Lo anterior, como ya se mencionó, va en contravía de la tendencia presentada en Bogotá, ya que los grupos tienden a tener mayor similitud en la distribución espacial.

Tabla 6. Indicador de Disimilitud en Bogotá por sector y localidad, años 2005 y 2018

\begin{tabular}{|l|r|r|r|r|}
\hline \multirow{2}{*}{ GRUPOS } & \multicolumn{2}{|c|}{ Sector } & \multicolumn{2}{c|}{ Comuna } \\
\cline { 2 - 5 } & $(\mathrm{D}(\mathrm{s})) \mathbf{2 0 0 5}$ & $(\mathrm{D}(\mathrm{s})) \mathbf{2 0 1 8}$ & $(\mathrm{D}(\mathrm{s})) \mathbf{2 0 0 5}$ & $(\mathrm{D}(\mathrm{s})) \mathbf{2 0 1 8}$ \\
\hline ICVB Bajo - ICVB Medio & 0,52 & 0,52 & 0,35 & 0,33 \\
\hline ICVB Bajo - ICVB Alto & 0,82 & 0,71 & 0,67 & 0,58 \\
\hline ICVB Medio - ICVB Alto & 0,62 & 0,44 & 0,54 & 0,34 \\
\hline
\end{tabular}

Elaboración propia

$\mathrm{Al}$ estimar la probabilidad de interacción entre los grupos, se obtiene que es mínima para los grupos con ICVB bajo y alto, ya que está por debajo del 0,1 a escala de sector en ambos años de estudio. A escala de comuna, el indicador pasa a estar por encima de 0.1 , lo cual indica que hay una mayor relación, pero sigue siendo un indicador bajo. Sin embargo, los indicadores de interacción del grupo con ICVB Medio con los otros dos, es mucho más alta que en Bogotá y se incrementa en el periodo de estudio.

Tabla 7. Indicador de Interacción en Medellín por sector y comuna, años 2005 y 2018

\begin{tabular}{|l|r|r|r|r|}
\hline \multirow{2}{*}{ GRUPOS } & \multicolumn{2}{|c|}{ Sector } & \multicolumn{2}{c|}{ Localidad } \\
\cline { 2 - 5 } & $(\mathrm{xPy}) \mathbf{2 0 0 5}$ & $(\mathrm{xPy}) \mathbf{2 0 1 8}$ & $(\mathrm{xPy}) 2005$ & $(\mathrm{xPy}) 2018$ \\
\hline ICVB Bajo - ICVB Medio & 0,32 & 0,31 & 0,38 & 0,45 \\
\hline ICVB Bajo - ICVB Alto & 0,05 & 0,05 & 0,11 & 0,13 \\
\hline ICVB Medio - ICVB Alto & 0,25 & 0,38 & 0,27 & 0,44 \\
\hline
\end{tabular}

Elaboración propia

En Cali los indicadores estimados demuestran que la incidencia de la segregación significativamente superior a la de Medellín, pero inferior a la que se presenta en Bogotá. Sin embargo, al igual que en Medellín, se identifica que los indicadores bajaron en el periodo 2005-2018.

El Indicador de Segregación para el año 2005 a escala de sector muestra que la segregación más alta es la de los grupos con ICVB alto $(0,73)$, los cuales presentan una mayor tendencia a la conformación de conglomerados. La segregación del grupo con ICVB bajo puede ser considerado igualmente como alta, con un valor de 0,64. Al igual que en Bogotá y Medellín, a una escala mayor, estos indicadores son más bajos, lo cual indica que hay una mayor incidencia de la segregación a escala de sector que a una escala mayor de ciudad.

Para el año 2018 los indicadores de segregación del grupo con ICVB alto disminuyen tanto a escala de sector como a escala de comuna, pasando a 0,65 y 0,52 respectivamente, lo cual lleva a la conclusión 
que se dio una distribución más extendida de este grupo en el territorio. En el grupo con ICVB bajo también se presenta una disminución de la segregación a escala de sector al pasar a 0,53, pero en la escala de comuna el indicador se mantiene estable.

Tabla 8. Indicador de Segregación en Medellín por sector y comunas, años 2005 y 2018

\begin{tabular}{|l|c|c|c|c|}
\hline \multirow{2}{*}{ GRUPO } & \multicolumn{2}{|c|}{ Sector } & \multicolumn{2}{c|}{ Comuna } \\
\cline { 2 - 5 } & IS(s) 2005 & IS(s) 2018 & IS(s) 2005 & IS(s) 2018 \\
\hline ICVB Bajo & 0,64 & 0,49 & 0,53 & 0,49 \\
\hline ICVB Medio & 0,48 & 0,38 & 0,41 & 0,35 \\
\hline ICVB Alto & 0,73 & 0,69 & 0,65 & 0,52 \\
\hline
\end{tabular}

Elaboración propia

El Indicador de disimilitud ajustado por la longitud de la frontera y la relación perímetro / superficie, aplicado en la aglomeración urbana de Cali, muestra que la mayor disimilitud se presenta entre el grupo con ICVB bajo e ICVB alto. Sin embargo, esta disimilitud disminuye en el periodo de análisis de 0,86 a 0,73 en la escala sectorial y de 0,63 a 0,60 en la escala de comuna. Lo anterior, al igual que en Medellín, es contrario a lo presentado en Bogotá, ya que los grupos tienden a tener mayor similitud en la distribución espacial.

Tabla 9. Indicador de Disimilitud en Bogotá por sector y localidad, años 2005 y 2018

\begin{tabular}{|l|r|r|r|r|}
\hline \multirow{2}{*}{ GRUPOS } & \multicolumn{2}{|c|}{ Sector } & \multicolumn{2}{c|}{ Comuna } \\
\cline { 2 - 5 } & $(\mathrm{D}(\mathrm{s})) \mathbf{2 0 0 5}$ & $(\mathrm{D}(\mathrm{s})) \mathbf{2 0 1 8}$ & $(\mathrm{D}(\mathrm{s})) \mathbf{2 0 0 5}$ & $(\mathrm{D}(\mathrm{s})) \mathbf{2 0 1 8}$ \\
\hline ICVB Bajo - ICVB Medio & 0,58 & 0,45 & 0,35 & 0,30 \\
\hline ICVB Bajo - ICVB Alto & 0,86 & 0,80 & 0,63 & 0,60 \\
\hline ICVB Medio - ICVB Alto & 0,62 & 0,61 & 0,63 & 0,48 \\
\hline
\end{tabular}

Elaboración propia

Al estimar la probabilidad de interacción entre los grupos, se obtiene que es mínima para los grupos con ICVB bajo y alto, ya que está por debajo del 0,1 a escala de sector en ambos años de estudio. A escala de comuna, el indicador pasa a estar por encima de 0,1 , lo cual indica que hay una mayor relación, pero sigue siendo un indicador bajo. Sin embargo, los indicadores de interacción del grupo con ICVB Medio con los otros dos, es mucho más alta que en Bogotá, aunque inferior que la de Medellín, y se incrementa en el periodo de estudio.

Tabla 10. Indicador de Interacción en Medellín por sector y comuna, años 2005 y 2018

\begin{tabular}{|l|r|r|r|r|}
\hline \multirow{2}{*}{ GRUPOS } & \multicolumn{2}{|c|}{ Sector } & \multicolumn{2}{c|}{ Localidad } \\
\cline { 2 - 5 } & $(\mathrm{xPy}) \mathbf{2 0 0 5}$ & $(\mathrm{xPy}) \mathbf{2 0 1 8}$ & $(\mathrm{xPy}) 2005$ & $(\mathrm{xPy}) 2018$ \\
\hline ICVB Bajo - ICVB Medio & 0,26 & 0,33 & 0,31 & 0,42 \\
\hline ICVB Bajo - ICVB Alto & 0,03 & 0,05 & 0,12 & 0,13 \\
\hline ICVB Medio - ICVB Alto & 0,11 & 0,26 & 0,31 & 0,34 \\
\hline
\end{tabular}

Elaboración propia

Al comparar los indicadores en el año 2018 a escala de sector, se identifica que Bogotá es la ciudad en la que el grupo con ICVB bajo presenta mayor segregación, seguido de Medellín y de Cali. Se concluye que el grupo con ICVB alto es que la mayor segregación presenta en Cali y en Bogotá, ya que tiene unos indicadores altos. Sin embargo, en Medellín la tendencia no es igual y este grupo tiende a tener una segregación significativamente más baja que en las otras tres ciudades.

Acorde con lo anterior, la mayor disimilitud entre los grupos según su ICVB se encuentra en Bogotá y Cali, principalmente entre los grupos bajo y alto. Está disimilitud, sin embargo, es significativamente menor en Medellín, ciudad en la cual en el periodo de análisis se dio un cambio en la distribución de los grupos en el espacio del conglomerado urbano. 
Figura 9. Comparación Segregación Bogotá, Cali y Medellín, año 2018 a escala de sector

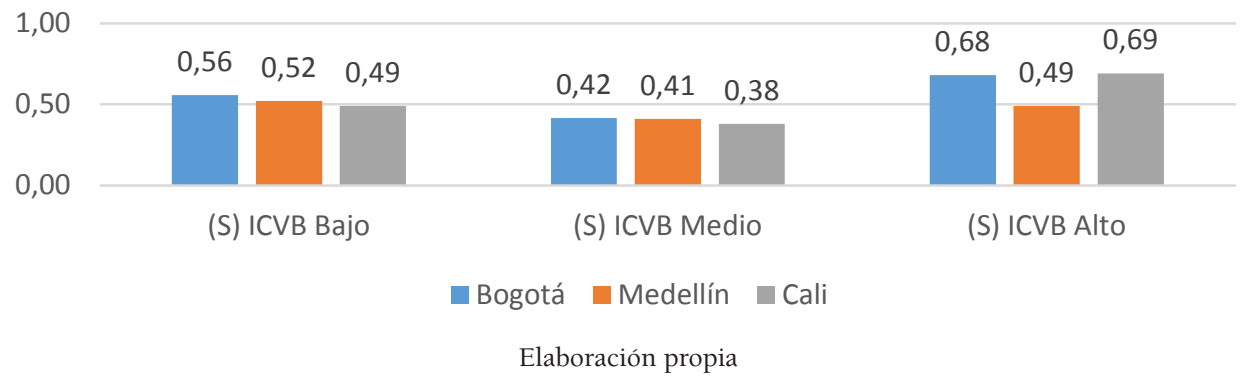

Figura 10. Comparación Disimilitud Bogotá, Cali y Medellín, año 2018 a escala de sector

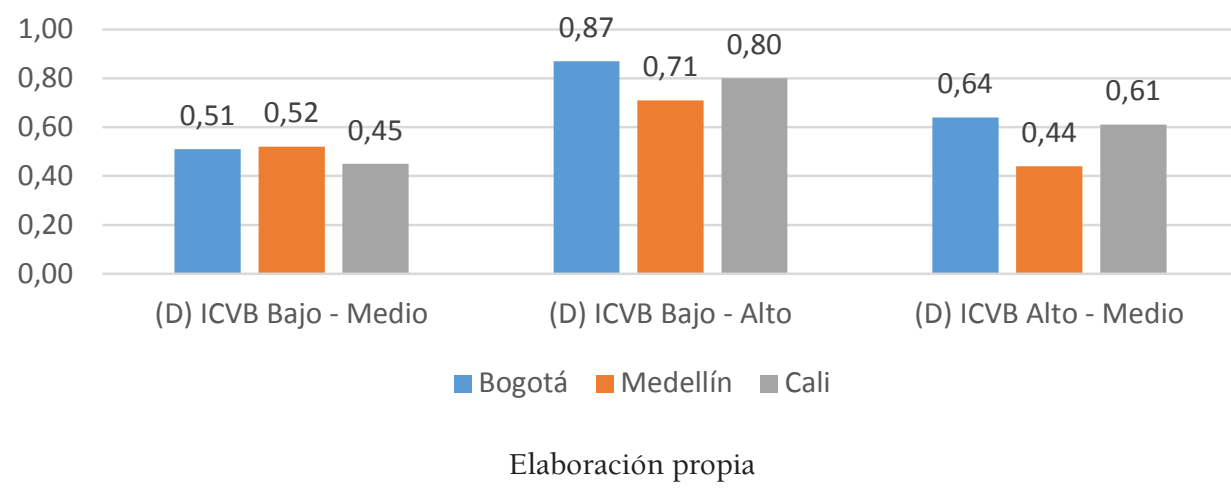

Por último, la interacción en las tres aglomeraciones indica que hay una baja probabilidad de interacción a escala de sector entre los grupos con ICVB bajo e ICVB alto. Si bien los indicadores anteriores muestran una diferencia de Medellín con respecto a Bogotá y Cali, en este caso las tres ciudades están en el mismo panorama.

Figura 11. Comparación Interacción en Bogotá, Cali y Medellín, año 2018 a escala de sector

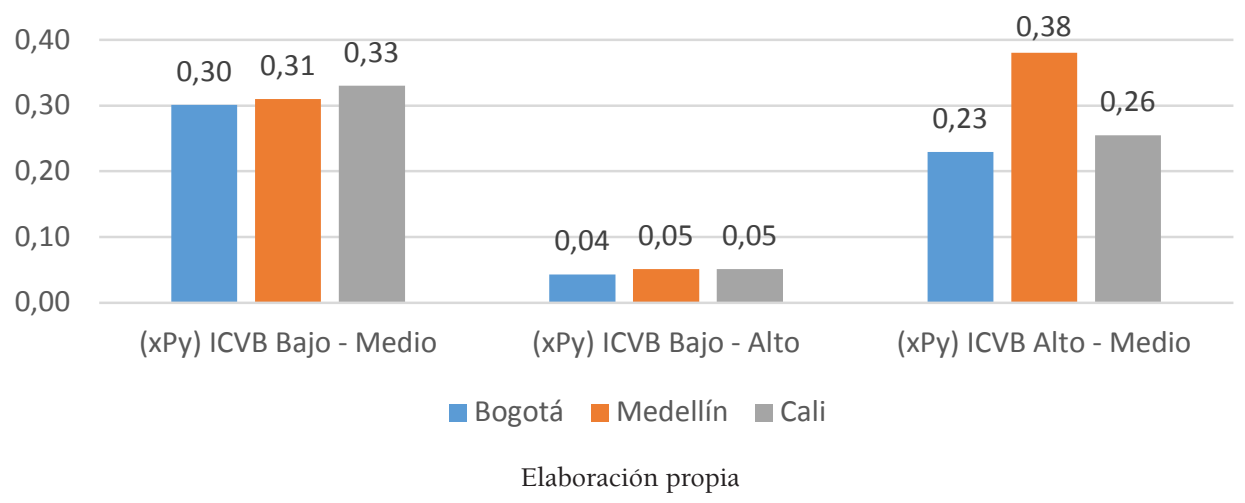

\section{Discusión de resultados}

La segregación residencial sigue siendo un fenómeno presente en las ciudades y por lo cual la investigación sobre la misma mantiene vigencia. El libro editado por Musterd (2020) da cuenta de un fenómeno con múltiples manifestaciones, múltiples interrelaciones y diferentes impactos, según el contexto en el que se investigue. Esto pone de manifiesto la importancia de aportar insumos, tanto globales como locales, para el debate sobre la segregación residencial, no solo en la academia, sino en el marco de la formulación de políticas públicas.

Con base en los resultados obtenidos se centra la discusión en la importancia de hacer análisis comparativos entre ciudades, dado que se pone de manifiesto que, aún en el mismo país, la segregación re- 
sidencial no es un fenómeno homogéneo y constante en el tiempo. Acorde a lo planteado por Maloutas (2012), los resultados indican que la segregación es un fenómeno con magnitud y tendencia espacial que varían en función del tiempo y las ciudades.

Si bien en las ciudades estudiadas hay una evidente segregación residencial, se pone en discusión que todas las metrópolis contemporáneas de Latinoamérica tengan los mismos patrones geográficos, como lo planteado desde Griffin y Ford (1980) hasta Borsdorf (2003), quienes manifiestan como un genérico la conformación de una cuña lineal de alta renta como uno de los elementos característicos de la segregación en región. Si bien este parece ser un elemento que comparte Bogotá con otras ciudades como Santiago de Chile, en el caso de Medellín y de Cali no es tan claro, lo cual indica que hay unos patrones diferentes de conformación espacial de la diferenciación social que responden a sus condiciones históricas, geográficas, culturales y políticas.

Dado que la segregación no es estática y presenta cambios, uno de los resultados obtenidos que merece discusión es la mayor incidencia de la segregación a escala de sector. Con la transformación de las ciudades y la aparición de nuevas tipologías residenciales como los conjuntos cerrados y los barrios isla, se ha presentado un proceso de compactación de las ciudades que produce cambios en la escala espacial de la segregación. Esto indica que los grupos, según su calidad de vida, tienden a aproximarse a una escala general de ciudad, pero se separan en los espacios más próximos de la vida cotidiana, como los barrios, por ejemplo. Esto va en concordancia a lo mostrado por Sabatini et al. (2012) y Rasse (2015), en las que se muestra cómo los patrones geográficos de la segregación tienden a la disminución de la escala en las ciudades latinoamericanas, tal como se presenta en Bogotá, Medellín y Cali, en donde la segregación es mayor a escala barrial.

Es importante destacar que, según los resultados obtenidos, los grupos con ICVB alto presentan una mayor segregación espacial en las tres aglomeraciones estudiadas. Teniendo en cuenta la discusión conceptual al respecto de la influencia del mercado del suelo hecha por Harding (2014) y Ospino (2017), se plantea que la generación de zonas homogéneas socialmente y la aparición de nuevas tipologías edificatorias que permiten la consolidación de barrios "cerrados", que les consienten a los grupos altos mantenerse separados de los demás.

La segregación de los grupos altos supone un impacto fuerte en la posibilidad de interacción que se puede dar en la ciudad. La falta de interacción entre grupos supone un impacto principal para quienes se encuentran en una situación negativa, dado que se limitan sus interacciones de la vida cotidiana a quienes tienen sus mismas condiciones y posibilidades (Mayorga, 2012). Así, se minimizan las posibilidades de sostener interacciones con otros individuos que estén en posibilidades de suministrar información sobre fuentes de trabajo, capacitación, negocios, oportunidades educativas, etc.

Lo anterior se refuerza, si se tiene en cuenta que la uniformidad social de un lugar y las deficiencias en el acceso a las dinámicas urbanas, como una de las características identificables del fenómeno de la segregación de los grupos localizados en la parte baja de la estructura social, dista de ser un producto de la agregación de las decisiones libres de localización (Loaiza y Carvajal, 2014). Más bien, está directamente vinculada a la dificultad de acceder a suelo barato donde producir procesos de urbanización con viviendas adecuadas y dotaciones suficientes (Mier, et al. 2012).

En términos metodológicos, resulta importante resaltar el potencial de la aplicación de técnicas estadísticas y de análisis espacial como complemento para el estudio de la segregación residencial, pues en la medida en que se realicen análisis a diversas escalas será posible obtener una aproximación más precisa de su comportamiento. Al respecto, lograr mediciones del fenómeno en unidades más ajustadas a la heterogeneidad de un territorio, permite la estimación de modelos de medición estadística que indiquen la relación compleja entre variables espaciales y variables socioeconómicas. Esto va en línea por lo planteado por Ortiz y Escolano (2013) al respecto de reflexionar sobre el problema de la unidad espacial modificable en el estudio de la segregación residencial, que los resultados pueden variar en función de la desagregación o agrupación espacial de las observaciones. Igualmente es importante establecer a partir de los indicadores cómo se da el potencial aislamiento o interacción por contigüidad o cercanía (Garroncho y Campos-Alanis 2013).

La investigación realizada supone un avance en tres aspectos metodológicos: 1) la identificación de la unidad espacial de análisis, 2) la clasificación de los grupos sociales y 3) la caracterización de los indicadores espaciales de segregación más adecuados (Rodríguez, 2013). En el primer punto, por ejemplo, en 
las investigaciones realizadas por la Secretaria Distrital de Planeación (2007 y 2013) al no tener en cuenta la escala de las observaciones desconoce las implicaciones estadísticas sobre la desviación al seleccionar datos previamente agrupados. En términos generales, en la investigación hecha en Colombia se utilizan modelos que no tienen en cuenta la configuración y la escala de las unidades espaciales (Mayorga, 2019). En el segundo punto, sobre la clasificación de los grupos sociales, en el caso de Salas (2008) al usar como clasificación datos unidimensionales de educación de la población, Aliaga y Álvarez (2010) al tomar la estratificación oficial y Dureau, et al. (2015) al utilizar tres variables para su ICS, asumen medidas preestablecidas o unidimensionales que no permiten establecer la diferencia. En el tercer aspecto, ninguna de las investigaciones reseñadas por Mayorga (2019) se utilizan indicadores de segregación que contengan variables espaciales, se utilizan las medidas clásicas de segregación que no tienen en cuenta la configuración espacial.

\section{Conclusiones}

A partir de los análisis realizados sobre los cambios en los patrones e indicadores de segregación en las principales aglomeraciones del sistema urbano de Colombia, se identifica que, en los dos años de corte y las dos escalas analizadas, hay una mayor incidencia del fenómeno en Bogotá. Si bien se podría afirmar que la segregación se incrementa con la magnitud poblacional de las aglomeraciones urbanas, esta hipótesis no se cumple con los resultados de Medellín y Cali, dado que la segunda es una aglomeración menor en términos poblacionales, pero la incidencia de la segregación es significativamente superior.

Con respecto a la temporalidad, los resultados indican que solo en el caso de Bogotá los indicadores estimados tienden a incrementarse entre el año 2005 y 2018. Tanto en Medellín como en Cali, los indicadores tienden a disminuirse, lo cual abre nuevas preguntas sobre las condiciones particulares de estas ciudades en el contexto de su ordenamiento territorial, el mercado inmobiliario de vivienda y la ejecución de políticas públicas.

Por último, se destaca de los resultados que el mayor problema en términos de segregación se evidencia al analizar la disimilitud y la integración, dado que en las tres ciudades los indicadores muestran que los grupos extremos tienden a no mezclarse a escala sectorial o escala de ciudad. De tal forma, si bien los indicadores de segregación del grupo con ICVB bajo no son altos en las tres ciudades, la disimilitud y la interacción muestran que su distribución espacial conforma conglomerados que refuerzan sus condiciones de vida bajas.

En términos de las ciudades estudiadas, se concluye y resalta la importancia de realizar estudios que contemplen la complejidad territorial y no se limiten a la delimitación político-administrativa para realizar estudios sobre segregación. De allí la importancia de realizar apuestas que permitan la comprensión del fenómeno de segregación incluyendo los municipios secundarios que tienen una relación funcional y territorial con la ciudad principal, de manera tal que se puedan dar resultados más objetivos sobre el fenómeno.

Sobre la base de los resultados, teniendo en cuenta que el ICVB se puede replicar para todas las ciudades de Colombia, tanto para el año 2005 y el 2018, se plantea la posibilidad de estimar indicadores de segregación residencial y su cambio en el periodo para para las 17 aglomeraciones urbanas identificadas por DNP (2014). Esto implicaría tener información sobre segregación residencial para el 4\% de lo municipios del país, pero en ellos se concentra el $51 \%$ del total de la población, lo cual supone un aporte sustancial para el diseño de una política urbana que afronte los impactos negativos del fenómeno, especialmente entre los grupos con mayores privaciones.

Se sobre la relevancia de hacer una comparación del fenómeno entre distintas ciudades, dado que esto permite observar de manera diferenciada las expresiones territoriales de la segregación con otros países y continentes. Acorde con lo hecho por Dureau, et al. (2015) al comparar Bogotá, Santiago y Sao Paulo, se abren líneas para comparar la segregación residencial en las ciudades colombianas con otros contextos, involucrando aspectos que no se han tenido en cuenta, como las recientes migraciones que se están dando en el sur del continente.

\section{Referencias}

Aguilar, A. (2002). Las mega-ciudades y las periferias expandidas. EURE (Santiago), 28(85), 121-149. https://dx.doi.org/10.4067/S0250-71612002008500007 
Alfonso, O.A. (2017). La calidad de Bogotá en perspectiva comparada: urbanismo, simbolismos y segregación. En O.A. Alfonso Roa (Ed.), Bogotá en la Encrucijada del Desorden (pp. 27-82). Bogotá: Universidad Externado de Colombia.

Aliaga, L. y Álvarez , M. (2010). Segregación residencial en Bogotá a través del tiempo y diferentes escalas. Documento de Trabajo de Lincoln Institute of Land Policy. Recuperado de http://www. institutodeestudiosurbanos.info/eventos/seminarios-de-investigacion-urbano-regional-aciur/ memorias-viii-seminario-aciur-2009/mesas-tematicas/fragmentacion-apropiacion-y-regulacion/368segregacion-residencial-en-bogota-a-traves-del-tiempo-y-a-distintas-escalas/file

Artigas, A., Chabalgoity, M., García, A., Medina, M. y Trinchitella, J. (2002). Transformaciones socioterritoriales del área metropolitana de Montevideo. EURE (Santiago), 28(85), 151-170. https://dx.doi. org/10.4067/S0250-71612002008500008

Barh, J. y Mertins, G. (1982). A model of the social and spatial differentiation of latin American metropolitan cities. Applied Geography and development, 19, 22-45.

Borsdorf, A. (2003). Cómo modelar el desarrollo y la dinámica de la ciudad latinoamericana. EURE (Santiago), 29(86), 37-49. https://dx.doi.org/10.4067/S0250-71612003008600002

Carialo, C. y Locabana, M. (2001). La metrópoli fragmentada. Caracas entre la pobreza y la globalización. EURE (Santiago), 27(80). https://dx.doi.org/10.4067/S0250-71612001008000002

Carrasco Bayona, J. (2007). La segregación residencial de la población extranjera en Barcelona: ¿una segregación fragmentada?. Revista Electrónoca de Geografía y Ciencias Sociales, XI(235). Recuperado de https://revistes.ub.edu/index.php/ScriptaNova/article/view/1309.

De Esteban, A., Curiel Díaz, Javier, \& Perello, Salvador. (2003). Inmigración y segregación urbana. Papeles de economía española, 98. Recuperado de https://dialnet.unirioja.es/servlet/articulo? codigo=786562

Departamento Administrativo Nacional de Estadística (DANE) (2005). Datos del Censo Nacional de población y Vivienda. Recuperado de http://systema59.dane.gov.co/bincol/rpwebengine.exe/ PortalAction?lang=esp

Departamento Administrativo Nacional de Estadística (DANE) (2018). Datos del Censo Nacional de población y vivienda. Recuperado de http://systema59.dane.gov.co/bincol/rpwebengine.exe/ PortalAction?lang=esp

Departamento Administrativo Nacional de Estadística (DANE) (2020). Proyecciones poblacionales. Recuperado de https://www.dane.gov.co/index.php/estadisticas-por-tema/demografia-y-poblacion/ proyecciones-de-poblacion

Departamento Nacional de Planeación (DNP) (2014). Sistema de Ciudades, una aproximación al caso colombiano. Bogotá, DNP.

Dureau, F, Contreras, Y., Cymbalista, R., Le Reux, G. y Piron, M. (2015). Evolución de la intensidad y de las escalas de la segregación residencial desde los años 1990: un análisis comparativo. En, F. Dureau (Coord.). Movilidades y cambio urbano Bogotá, Santiago y Sao Paulo, 127-156. Bogotá: Universidad Externado de Colombia.

Ford, L. (1996). A New and Improved Model of Latin American City Structure. Geographical Review, 86(3), 437-440. https://doi.org/10.2307/215506

Garroncho, C. y Campos-Alanís, J. (2013). Réquiem por los indicadores no espaciales de segregación residencial. Papeles de Población, 19(77), 269-300. Recuperado de http://www.scielo.org.mx/pdf/pp/ v19n77/v19n77a14.pdf

Gormsen, E. (1991). Urban rehabilitation of historic town centres in Latin America. Applied Geography, 38, 22-39.

Griffin, E. y Ford, L. (1980). A model of Latin American city structure. Geographical Review, 70(4), $397-422$. https://doi.org/10.2307/214076

Harding, A. (2014). Urban Theory: A critical introduction to power, cities and urbanism in the 21st century. SAGE Publications

Janoschka, M. (2002). El nuevo modelo de la ciudad latinoamericana: fragmentación y privatización. EURE (Santiago), 28(85), 11-20. https://dx.doi.org/10.4067/S0250-71612002008500002 
Jargowsky, P. (1997). Poverty and Place; guettos, barrios, and the american city. New York: Ruseel Sage Foundation.

Katzman, R. (2005). Segregación espacial, empleo y pobreza en Montevideo. Revista de la Cepal, abril, 131-148. https://doi.org/10.18356/93a498f9-es

León, N. y Ruiz, C. (2016). EL sistema urbano en Colombia y la formación metropolitana: una aproximación desde la Nueva Geografía Económica. Cuadernos de Geografía: Revista Colombiana de Geografía, 25(2), 21-37. http://dx.doi.org/10.15446/rcdg.v25n2.52850

León, N. y Ruiz, C. (2016). El sistema urbano en Colombia y la formación metropolitana: una aproximación desde la Nueva Geografía Económica. Cuadernos de Geografía: Revista Colombiana de Geografía, 25(2), 21-37. https://doi.org/10.15446/rcdg.v25n2.52850

Loaiza Cerón, W. y Carvajal Escobar, Y. (2014). Índice de segregación espacial y socioeconómico (ISES) en las comunas de Santiago de Cali. Cuadernos de Vivienda y Urbanismo, 7(13), 84-101. https://doi. org/10.11144/Javeriana.CVU7-13.ises

Maloutas, T. (2012). Residential Segregation in Context. En Maloutas, T y Fujita, (ed). Comparative Perspective Making Sense of Contextual Diversity. Routdlege.

Marengo, C., y Elorza, A. (2014). Tendencias de segregación residencial socioeconómica: el caso de Córdoba (Argentina) en el período 2001-2008. EURE (Santiago), 40(120), 111-113. https://dx.doi. org/10.4067/S0250-71612014000200006

Martori, J., Hoberg, K. y Surinach, J. (2006). Población inmigrante y espacio urbano. Indicadores de segregación y pautas de localización. EURE (Santiago), 32(97), 49-62. https://dx.doi.org/10.4067/ $\underline{\text { S0250-71612006000300004 }}$

Massey, D. y Denton, N. (1988). The dimensions of residential segregation. Social Forces, 67(2), 281-315. https://doi.org/10.2307/2579183

Mavrou, I. (2015). Análisis Factorial Exploratorio: Cuestiones conceptuales y metodológicas. Revista Nebrija de Lingüistica Aplicada a la Enseñanza de Lenguas, 19, 71-80. https://doi.org/10.26378/ $\underline{\text { nnlael019283 }}$

Mayorga, J. (2012). Capital Social, Segregación y equipamientos Colectivos. Revista Darq, 11, 22-31. https://doi.org/10.18389/dearq11.2012.04

Mayorga, J., García, D. y Hernández, L. (2017). Calidad de vida y su correlación con los precios del suelo: aproximación a la segregación residencial en Bogotá. Cuadernos de vivienda y urbanismo, 10(19), 22-40. https://doi.org/10.11144/Javeriana.cvu10-19.cvcp

Mayorga, J. (2019). Una revisión de la investigación sobre segregación urbana en Colombia: una lectura crítica desde la geografía. Revista Ciudades, Estados y Política, 6(2), 36-5. https://doi.org/10.15446/ cep.v6n2.83993

Mayorga, J., García, D. y Barrera, R.(2019). Cálculo de un indicador de calidad de vida básico para Bogotá por secciones censales mediante análisis factorial. Perspectiva Geográfica, 24(1), 53-74. https://doi. org/10.19053/01233769.7861

Medina, C., Morales, L. y Núñez, J. (2008). Quality of Life in Urban Neighborhoods in Colombia: The Cases of Bogotá and Medellín. Borradores de Economía, 536. Recuperado de https://investiga.banrep. gov.co/es/content/quality-life-urban-neighborhoods-colombia-cases-bogota-and-medellin

Merengo, C. y Elorza, A. (2014). Tendencias de segregación residencial socioeconómica: el caso de Córdoba (Argentina) en el período 2001-2008. EURE (Santiago), 40(120), 111-113. https://dx.doi. org/10.4067/S0250-71612014000200006

Mier, A., Velásquez, I. y Zicardi, A. (2012). Pobreza urbana, segregación residencial y mejoramiento del espacio público en la ciudad de México. Sociologías, Porto Alegre, 30, 118-155. https://doi.org/10.1590/ $\underline{\text { S1517-45222012000200005 }}$

Montoya Gray, J. W. (2013). El sistema urbano colombiano frente a la globalización: reestructuración económica y cambio regional. Cuadernos de vivienda y urbanismo, 6(12), 302-320. Recuperado de https://revistas.javeriana.edu.co/index.php/cvyu/article/view/7038 
Montoya, J. (2013). El sistema urbano colombiano frente a la globalización: reestructuración económica y cambio regional. Cuadernos de vivienda y urbanismo, 6(12) , 302-320. https://doi.org/10.11144/ Javeriana.cvu6-12.sucf

Montoya, O. (2007). Aplicación del análisis factorial a la investigación de mercados. Caso de estudio. Scientia et Technic, Año XIII, 35. Universidad Tecnológica de Pereira.

Musterd, S. (2020). Urban segregation: contexts, domains, dimensions and approaches. En S. Musterd (Ed), Handbook of Urban Segregation. Elga. https://doi.org/10.4337/9781788115605.00007

Ortiz, J. y Escolano, S. (2013). Movilidad residencial del sector de renta alta del Gran Santiago (Chile): hacia el aumento de la complejidad de los patrones socio espaciales de segregación. EURE (Santiago), 39(118), 77-96. https://dx.doi.org/10.4067/S0250-71612013000300004

Ortiz, J. y Schiappacasse, P. (2000). Evolución de la diferenciación areal interna del espacio social del Gran Santiago: una dinámica opuesta a la sostenibilidad social de la ciudad. Investigaciones Geográficas Chile, 34, 61-76. https://doi.org/10.5354/0719-5370.2000.27732

Ospino. N. (2017). Building the Inclusive City: Theory and Practice for Confronting Urban Segregation. Routdlege.

Paradis, E. (2009). Moran's autocorrelation coefficient in comparative methods. R Foundation for Statistical Computing, Vienna. Recuperado de https://cran.r-project.org/web/packages/ape/vignettes/ MoranI.pdf

Park, R. (1999). La ciudad y otros ensayos de ecología urbana. Barcelona: Ediciones Serbal.

Prevot, M. (2002). Buenos Aires en los años 90: metropolización y desigualdades. EURE (Santiago), 28(85), 31-50. https://dx.doi.org/10.4067/S0250-71612002008500003

Rasse, A. (2015). Juntos, pero no revueltos. Procesos de integración social en fronteras residenciales entre hogares de distinto nivel socioeconómicol. EURE (Santiago), 41(122), 125-143. https://dx.doi. org/10.4067/S0250-71612015000100006

Rodríguez, G. (2013). El uso de zonas censales para medir la segregación residencial. Contradicciones, propuesta metodológica y un estudio de caso: Argentina 1991-2001. EURE (Santiago), 39 (118), 97-122. https://dx.doi.org/10.4067/S0250-71612013000300005

Rodríguez, G. (2014). Qué es y qué no es segregación residencial. Contribuciones para un debate pendiente. Biblio 3W. Revista Bibliográfica de Geografía y Ciencias Sociales, 19(1079). Recuperado de http://www.ub.edu/geocrit/b3w-1079.htm

Rodríguez, J. y Arriagada, C. (2004). Segregación Residencial en la Ciudad Latinoamericana. EURE (Santiago), 30, 5-24. http://dx.doi.org/10.4067/S0250-71612004008900001

Ruiz, J. y López, E. (2014). El estudio de la segregación residencial en Santiago de Chile: revisión crítica de algunos problemas metodológicos y conceptuales. EURE (Santiago), 40(119), 25-48. https://dx.doi. org/10.4067/S0250-71612014000100002

Sabatini, F. (2000). Reforma de los mercados de suelo en Santiago, Chile: efectos sobre los precios de la tierra y la segregación residencial. EURE (Santiago), 23(77), 49-80. https://doi.org/10.4067/S0250$\underline{71612000007700003}$

Sabatini, F. (2006). La segregación social del espacio en las ciudades de América Latina. Banco Interamericano de Desarrollo.

Sabatini, F., Rasse, A., Mora, P. y Brain, I. (2012). ¿Es posible la integración residencial en las ciudades chilenas? Disposición de los grupos medios y altos a la integración con grupos de extracción popular. EURE (Santiago), 38(115), 159-194. https://dx.doi.org/10.4067/S0250-71612012000300008

Salas, A. (2008). Residential segregation and housing production in Bogota, between perceptions and realities. Tesis para optar al título de Doctora en Geografia. Universidad de Poitiers, Departamento de Geografía.

Secretaría Distrital de Planeación \& Universidad Nacional de Colombia (2007). Segregación Socioeconómica en el espacio urbano de Bogotá. Bogotá, CID-Universidad Nacional de Colombia.

Secretaría Distrital de Planeación \& Universidad Nacional de Colombia (2013). Segregación Socioeconómica en el espacio urbano de Bogotá. Bogotá, CID-Universidad Nacional de Colombia. 
Secretaría Distrital de Planeación (SDP) (2007). Segregación Socioeconómica en el espacio urbano de Bogotá. Bogotá: CID-Universidad Nacional de Colombia.

Vallejo, P. M. (2013). El Análisis Factorial en la construcción e interpretación de tests, escalas y cuestionarios. Madrid: Universidad Pontificia Comillas.

Vázquez, L. (2018). Segregación Residencial en Montevideo:¿ Cuál fue su evolución en un contexto de recuperación económica, mejoras distributivas y crecimiento del ingreso real (Tesis de Maestría en Economía. Facultad de Ciencias Económicas y Administración). Recuperado de https://docplayer. es/94394269-Segregacion-residencial-en-montevideo.html

Velásquez, C. (2012). Vivienda social y ordenamiento territorial en Medellín durante el periodo 20062011: Pasos hacia la segregación residencial. Revista Territorios, (27), 181-197. Recuperado de https:// www.redalyc.org/pdf/357/35725869004.pdf

Vivas, H. (2013). Persistence of the residential segregation and composition of the human capital For neighborhoods in the city of Cali. Ensayos sobre politica económica, 31. Recuperado de http://www. scielo.org.co/scielo.php?script=sci_arttext $\&$ pid=S0120-44832013000100004\&lng=en\&tlng=es

White, M. (1983). The measurement of spatial segregation. The American Journal of Sociology, 88(5), 1008-1018. https://doi.org/10.1086/227768

Yong, An Gie, \& Pearce, Sean. (2013). A beginner's guide to factor analysis: Focusing on exploratory factor analysis. Tutorials in quantitative methods for psychology, 9(2), 79-94. https://dx.doi.org/10.20982/ tqmp.09.2.p079 